\title{
SBV regularity of entropy solutions for a class of genuinely nonlinear scalar balance laws with non-convex flux function
}

\author{
Robyr, $\mathrm{R}$
}

\begin{abstract}
In this work we study the regularity of entropy solutions of the genuinely nonlinear scalar balance laws We assume that the source term g $\mathrm{C} 1(x \times+)$, that the flux function $\mathrm{f} C 2(\times x+)$ and that ui $\quad$ fuu $(u i, x, t)=0$ is at most countable for every fixed $(x, t) \quad \Omega$. Our main result, which is a unification of two proposed intermediate theorems, states that BV entropy solutions of such equations belong to $\operatorname{SBVloc}(\Omega)$. Moreover, using the theory of generalized characteristics we prove that for entropy solutions of balance laws with convex flux function, there exists a constant $\mathrm{C}>0$ such that: where $\mathrm{C}$ can be chosen uniformly for $(\mathrm{x}+\mathrm{h}, \mathrm{t}),(\mathrm{x}, \mathrm{t})$ in any compact subset of $\Omega$.
\end{abstract}

DOI: https://doi.org/10.1142/S0219891608001544

Posted at the Zurich Open Repository and Archive, University of Zurich ZORA URL: https://doi.org/10.5167/uzh-8522

Journal Article

Accepted Version

Originally published at:

Robyr, R (2008). SBV regularity of entropy solutions for a class of genuinely nonlinear scalar balance laws with non-convex flux function. Journal of Hyperbolic Differential Equations, 5(2):449-475.

DOI: https://doi.org/10.1142/S0219891608001544 


\title{
SBV regularity of entropy solutions for a class of genuinely nonlinear scalar balance laws with non-convex flux function.
}

\author{
R.Robyr - UNI Zurich - Preprint
}

January 17,2008

\begin{abstract}
In this work we study the regularity of entropy solutions of the genuinely nonlinear scalar balance laws$$
D_{t} u(x, t)+D_{x}[f(u(x, t), x, t)]+g(u(x, t), x, t)=0 \quad \text { in an open set } \Omega \subset \mathbb{R}^{2} .
$$

We assume that the source term $g \in C^{1}\left(\mathbb{R} \times \mathbb{R} \times \mathbb{R}^{+}\right)$, that the flux function $f \in$ $C^{2}\left(\mathbb{R} \times \mathbb{R} \times \mathbb{R}^{+}\right)$and that $\left\{u_{i} \in \mathbb{R}: f_{u u}\left(u_{i}, x, t\right)=0\right\}$ is at most countable for every fixed $(x, t) \in \Omega$. Our main result, which is a unification of two proposed intermediate theorems, states that $B V$ entropy solutions of such equations belong to $S B V_{l o c}(\Omega)$. Moreover, using the theory of generalized characteristics we prove that for entropy solutions of balance laws with convex flux function, there exists a constant $C>0$ such that:

$$
u([x+h]+, t)-u(x-, t) \leq C h, \quad(h>0)
$$
\end{abstract}

where $C$ can be chosen uniformly for $(x+h, t),(x, t)$ in any compact subset of $\Omega$.

\section{Introduction}

In [2] the authors have shown that entropy solutions $u(x, t)$ of scalar conservation laws

$$
D_{t} u(x, t)+D_{x}[f(u(x, t))]=0 \quad \text { in an open set } \Omega \subset \mathbb{R}^{2}
$$

with locally uniformly convex flux function $f \in C^{2}(\mathbb{R})$ and $f^{\prime \prime}>0$, are functions of locally special bounded variation, i.e. the distributional derivative $D u$ has no Cantor part. In the proof proposed by Ambrosio and De Lellis the good geometric structure of the characteristics field correlated to the entropy solution play an important role and allows to define a geometric functional which jumps every time when a Cantor part of the distributional derivative $D u(., t)$ appears in the solution. In particular we recall here two significant properties of the characteristics: they are straight lines and two different 
backward characteristics can cross only at $t=0$ (the so-called no crossing property). We note also that for equations (1) we can take the well-known Oleinik estimate as entropy criterion, i.e. a distributional solution $u(x, t)$ of $(1)$ is an entropy solution provided that:

$$
u(x+z, t)-u(x, t) \leq \frac{\breve{C}}{t} z, \quad \text { for a } C>0
$$

holds for all $t>0, x, z \in \mathbb{R}$ where $z>0$. In [2] the one-sided estimate (2) is used as entropy criterion and it is used to get the proof.

In this note we extend this regularity result to a bigger class of hyperbolic conservation laws. At first we again consider scalar conservation laws (1) but allowing the change of convexity of the flux function $f$ at a countable set of points. One of the difficulties in dealing with these equations is that rarefaction waves may appear even for $t>0$ and consequently the no crossing property used in [2] does not hold. For instance, it is possible to construct a Riemann problem where the flux function has two inflections points and a shock splits into two contact discontinuities (see [11]). As we will see the strategy of the proof is not as complicated as one can expect: using an appropriate covering of $\Omega$ and working locally we reduce the problem to the convex or concave case. Thus, our first extension theorem states:

Theorem 1.1. Let $f \in C^{2}(\mathbb{R})$ be a flux function, such that $\left\{u_{i} \in \mathbb{R}: f^{\prime \prime}\left(u_{i}\right)=0\right\}$ is at most countable. Let $u \in B V(\Omega)$ be an entropy solution of the scalar conservation law (1). Then there exists a set $S \subset \mathbb{R}$ at most countable such that $\forall \tau \in \mathbb{R} \backslash S$ the following holds:

$$
u(., \tau) \in S B V_{l o c}\left(\Omega_{\tau}\right) \quad \text { with } \Omega_{\tau}:=\{x \in \mathbb{R}:(x, \tau) \in \Omega\} .
$$

In the second part of this paper we focus our attention on genuinely nonlinear scalar balance laws

$$
D_{t} u(x, t)+D_{x}[f(u(x, t), x, t)]+g(u(x, t), x, t)=0 \quad \text { in } \Omega \subset \mathbb{R}^{2}
$$

where the source term $g$ belongs to $C^{1}\left(\mathbb{R} \times \mathbb{R} \times \mathbb{R}^{+}\right)$, the flux functions $f$ belongs to $C^{2}\left(\mathbb{R} \times \mathbb{R} \times \mathbb{R}^{+}\right)$and $f_{\text {uи }}(., x, t)>0$ for any fixed $(x, t) \in \Omega$. Again the geometric structure of the characteristics is not as easy as in the case treated in [2]: now the characteristics are Lipschitz curves and in general are not straight lines. The different shape of the characteristics are due to the presence of the source term and to the dependence of $f$ on the points $(x, t) \in \Omega$. Fortunately, we can make use of the theory of generalized characteristics introduced by Dafermos (see [6],[7],[8]) to analyze the behavior of the characteristics for entropy solutions of (4). Important for our analysis is the no-crossing property between genuine characteristics. Thanks to this property we can expect to reproduce the geometric proof proposed in [2]. All the definitions and propositions about the theory of generalized characteristics, which are helpful in our work, are listed in section 3. Another problem, due to the presence of the source term and of the $(x, t)$ dependence, is that for equations $(4)$ the Oleinik estimate (2) stop to be true. Moreover, the Oleinik estimate cannot be taken as entropy criterion. What we can do, is to find a suitable generalization of this estimate, i.e. we will prove using the generalized characteristics that: 
Theorem 1.2. Let $f \in C^{2}\left(\mathbb{R} \times \mathbb{R} \times \mathbb{R}^{+}\right)$be a flux function such that $f_{\text {uu }}()>$.0 . Let $g \in C^{1}\left(\mathbb{R} \times \mathbb{R} \times \mathbb{R}^{+}\right)$be a source term and let $u \in L^{\infty}(\Omega)$ be an entropy solution of the balance law (4). In any fixed compact set $K \subset \Omega$ there exists a positive constant $C>0$ such that:

$$
u([x+z]+, t)-u(x-, t) \leq C z, \quad(z>0) .
$$

for every $(x, t),(x+z, t) \in K$.

However, for balance laws it is impossible to recover a constant of the form $C=\breve{C} / t$, where $C$ depends only on the time and on the second derivative of $f$, estimate (5) is sufficient to obtain all the regularity-results stated in this paper. The second theorem on the $S B V$ regularity proposed is:

Theorem 1.3. Let $f \in C^{2}\left(\mathbb{R} \times \mathbb{R} \times \mathbb{R}^{+}\right)$be a flux function such that $f_{\text {uu }}()>$.0 . Let $g \in C^{1}\left(\mathbb{R} \times \mathbb{R} \times \mathbb{R}^{+}\right)$be a source term and let $u \in L^{\infty}(\Omega)$ be an entropy solution of the balance law (4). Then there exists a set $S \subset \mathbb{R}$ at most countable such that $\forall \tau \in \mathbb{R} \backslash S$ the following holds:

$$
u(., \tau) \in S B V_{l o c}\left(\Omega_{\tau}\right) \quad \text { with } \Omega_{\tau}:=\{x \in \mathbb{R}:(x, \tau) \in \Omega\}
$$

Combining the two Theorems on the $S B V$ regularity we get a generalized Theorem, which says that also for balance laws with a flux function which changes convexity at most countable many times, the entropy solution is a locally $S B V$ function. Thus, as a consequence of Theorem 1.3 and 1.1 and of the slicing theory of $B V$ functions, we state:

Theorem 1.4. Let $f \in C^{2}\left(\mathbb{R} \times \mathbb{R} \times \mathbb{R}^{+}\right)$be a flux function, such that

$$
\left\{u_{i} \in \mathbb{R}: f_{u u}\left(u_{i}, x, t\right)=0\right\}
$$

is at most countable for any fixed $(x, t)$. Let $g \in C^{1}\left(\mathbb{R} \times \mathbb{R} \times \mathbb{R}^{+}\right)$be a source term and let $u \in B V(\Omega)$ be an entropy solution of the balance law (4):

$$
D_{t} u(x, t)+D_{x}[f(u(x, t), x, t)]+g(u(x, t), x, t)=0 \quad \text { in } \Omega \subset \mathbb{R}^{2} .
$$

Then there exists a set $S \subset \mathbb{R}$ at most countable such that $\forall \tau \in \mathbb{R} \backslash S$ the following holds:

$$
u(., \tau) \in S B V_{l o c}\left(\Omega_{\tau}\right) \quad \text { with } \Omega_{\tau}:=\{x \in \mathbb{R}:(x, \tau) \in \Omega\} .
$$

Moreover, $u(x, t) \in S B V_{l o c}(\Omega)$.

Scalar conservation laws in one space dimension and Hamilton-Jacobi equations in one dimension are strictly connected: entropy solutions correspond to viscosity solutions (see [9]). Thus, at the end of the paper using Theorem 1.3 we obtain also a regularity statement for viscosity solutions $u$ of a class of Hamilton-Jacobi equations: we prove that the gradient $D u$ of such solutions belongs to $S B V_{l o c}(\Omega)$. 
Corollary 1.1 (Hamilton-Jacobi). Let $H(u, x, t) \in C^{2}\left(\mathbb{R} \times \mathbb{R} \times \mathbb{R}^{+}\right)$be locally uniformly convex in $u$, i.e. $D_{u u} H>0$. If $w \in W^{1, \infty}(\Omega)$ is a viscosity solution of

$$
w_{t}(x, t)+H\left(w_{x}(x, t), x, t\right)=0,
$$

then $D w \in S B V_{l o c}(\Omega)$.

It would be interesting to find the same regularity for entropy $B V$ solutions of genuinely nonlinear system of conservation laws in one space dimension. We note that there are analogies between the structure of the generalized characteristics of systems and the one of the balance laws 4 of Theorem 1.4 proposed in here: in both cases the characteristics can intersect at $t \neq 0$ and in general they are not straight lines but Lipschitz curves, which are a.e. differentiable. Although the geometry of the characteristics field of these two problems seems to be similar, the case of systems looks much more difficult. Another open question is the local $S B V$ regularity for gradients of viscosity solutions of uniformly convex Hamilton-Jacobi PDEs in higher space dimensions. In [4] the authors have shown that under strong regularity assumptions on the initial functions $u_{0}$, the viscosity solution $u$ has a gradient $D u$, which belongs to the class $S B V$, i.e. $D^{2} u$ is a measure with no Cantor part (in fact the regularity theory of [4] and [5] gives stronger conclusions).

\section{Functions with bounded variation and special func- tions of bounded variation}

It is well-known that in general we cannot find classical smooth solutions for equations (1) and (4): shocks appear in finite time even for smooth initial data $u(x, 0)=u_{0}(x)$. In order to study all the possible solutions with jump discontinuities, we take the space of functions of bounded variation $B V$ as working space. We then collect some definitions and theorems about $B V$ and $S B V$ functions.

Definition 2.1. Let $u \in L^{1}(\Omega)$; we say that $u$ is a function of bounded variation in $\Omega$ if the distributional derivative of $u$, denoted by $D u$, is representable by a finite Radon measure on $\Omega$. A function $u \in L_{\text {loc }}^{1}(\Omega)$ has locally bounded variation in $\Omega$ if for each open set $V \subset \subset \Omega, u$ is a function of bounded variation in $V$. We write $u \in B V(\Omega)$ and $u \in B V_{l o c}(\Omega)$ respectively.

In our proofs we will have to deal with one-dimensional functions of bounded variation, therefore we collect here some useful properties. Using the Radon-Nikodym Theorem we split the Radon measure $D u$ into the absolute continuous part $D^{a} u$ (with respect to $\mathcal{L}^{1}$ ) and the singular part $D^{s} u$ :

$D u=D^{a} u+D^{s} u=D u\left\llcorner(\Omega \backslash S)+D u\left\llcorner S \quad\right.\right.$ where $S:=\left\{x \in \Omega: \lim _{\rho \downarrow 0} \frac{|D u|\left(B_{\rho}(x)\right)}{\rho}=\infty\right\}$. 
Let $A$ denote the set of atoms of $D u$, i.e. $x \in A$ if and only if $D u(\{x\}) \neq 0$. We recall that $A$ is at most countable. We now split the singular part $D^{s} u$ into the purely atomic part $D^{j} u$ and the diffusive part (i.e. without atoms) $D^{c} u$ :

$$
D u=D^{a} u+D^{s} u=D^{a} u+D^{j} u+D^{c} u=D u\llcorner(\Omega \backslash S)+D u\llcorner A+D u\llcorner(S \backslash A)
$$

where the two measures $D^{j} u$ and $D^{c} u$ are called respectively the jump and the Cantor part of the measure $D u$. The above decomposition is unique and the three measures $D^{a} u, D^{j} u, D^{c} u$ are mutually singular. Therefore we have $|D u|=\left|D^{a} u\right|+\left|D^{j} u\right|+\left|D^{c} u\right|$.

We are now ready to recall (see for instance Theorem 3.28 and Proposition 3.92 of [1]):

Proposition 2.1. Let $u \in B V(\Omega)$ and let $\Omega \subset \mathbb{R}$. Let $A$ be the set of atoms of Du. Then:

(i) Any good representative $\bar{u}$ is continuous in $\Omega \backslash A$ and has a jump discontinuity at any point of $A$. Moreover, $\bar{u}$ has classical left and right limits (denoted by $u^{L}$ and $u^{R}$ ) at any $x \in A$.

(ii) $D^{c} u$ vanishes on sets which are $\sigma$-finite with respect to $\mathcal{H}^{0}$ and on sets of the form $\bar{u}^{-1}(E)$ with $E \subset \mathbb{R}$ and $\mathcal{L}^{1}(E)=0$.

We conclude with:

Definition 2.2. Let $u \in B V(\Omega)$, then $u$ is a special function of bounded variation and we write $u \in S B V(\Omega)$ if $D^{c} u=0$, i.e. if the Cantor part is zero.

Remark 2.1. For similar definitions of $B V$ and $S B V$ functions in higher dimensions, we address the reader to Chapters 3 and 4 of [1].

\section{Entropy condition and generalized characteristics}

In this section, we will consider the balance laws (4) and we assume that $f:(u, x, t) \mapsto$ $f(u, x, t)$ and $g:(u, x, t) \mapsto g(u, x, t)$ are, respectively, $C^{2}$ and $C^{1}$ smooth functions on $\mathbb{R} \times \mathbb{R} \times \mathbb{R}^{+}$. Moreover, we prescribe that $f(., x, t)$ is strictly convex in $u$, i.e. $f_{u u}(., x, t)>0$ for fixed $(x, t)$. By the classical results on hyperbolic conservation laws it is known that with a suitable definition of weak solution and after the introduction of an admissibility condition, the so-called entropy condition, the existence and the uniqueness of an entropy solution of equations (1) and (4) are assured. Firstly we define the space of admissible solutions:

Definition 3.1. The entropy solution $u(x, t)$ of the equation (4) is a locally integrable function which satisfies the following properties:

1. For almost all $t \in[0, \infty)$ the one-sided limits $u(x+, t)$ and $u(x-, t)$ exist for all $x \in \mathbb{R}$.

2. $u(x, t)$ solves the balance equation (4) in the sense of distributions. 
3. For almost all $t \in[0, \infty)$ and for all $x \in \mathbb{R}$ we have that

$$
u(x-, t) \geq u(x+, t) .
$$

Throughout the paper we shall denote the entropy solution by $u(x, t)$ and we shall write $u(x+, t)$ and $u(x-, t)$ for the one-sided limits of $u(., t)$ (also denoted by $u^{R}$ and $u^{L}$ ). It is important to note that we will be dealing with entropy solutions $u(x, t)$, which belong to the space $B V_{l o c}(\Omega)$ and such that for every $t>0$ the function $u(., t)$ belongs to $B V_{l o c}(\mathbb{R})$. Moreover, we will restrict our analysis on good representative of solutions and then, under our initial hypothesis we follow the works of Dafermos $([6],[7],[8])$ giving an introduction to the theory of generalized characteristics and recalling here some results, which we shall use in the sequel.

Definition 3.2. A Lipschitz continuous curve $\chi(t)$, defined on an interval $I \subset \mathbb{R}^{+}$, is called a characteristic if it solves

$$
\dot{\chi}(t)=f_{u}(u(\chi(t), t), \chi(t), t)
$$

in the sense of Filippov [10], namely

$$
\dot{\chi}(t) \in\left[f_{u}(u(\chi(t)+, t), \chi(t), t), f_{u}(u(\chi(t)-, t), \chi(t), t)\right]
$$

for almost all $t \in I$.

By the theory of ordinary differential equations with discontinuous right-hand side like (12) (see [10]), we know that through every fixed point $(y, \tau) \in \mathbb{R} \times \mathbb{R}^{+}$passes at least one characteristic. We denote a characteristic either by $\chi(t)$ or by $\chi(t ; y, \tau)$ when the point $(y, \tau)$ must be specified. Every trajectory is confined between a maximal and a minimal characteristic (not necessarily distinct). Moreover, the speed of a generalized characteristic is not free and more precisely by Theorem 3.1 in [7] a characteristic either propagate at the classical speed or at shock speed:

Theorem 3.1. Let $\chi: I \rightarrow \mathbb{R}$ be a characteristic. Then for almost every $t \in I$

$$
\dot{\chi}(t)= \begin{cases}f_{u}(u(\chi(t) \pm, t), \chi(t), t), & \text { if } u(\chi(t)-, t)=u(\chi(t)+, t) ; \\ \frac{f(u(\chi(t)+, t), \chi(t), t)-f(u(\chi(t)-, t), \chi(t), t)}{u(\chi(t)+, t)-u(\chi(t)-, t)}, & \text { if } u(\chi(t)-, t)>u(\chi(t)+, t) .\end{cases}
$$

A backward (forward) characteristic trough any point $(y, \tau) \in \mathbb{R} \times \mathbb{R}^{+}$, is a characteristic $\chi$ defined on $[0, \tau]$ (respectively $[\tau, \infty))$ with $\chi(\tau)=y$. We call genuine a characteristic $\chi(t)$ such that $u(\chi(t), t)=u(\chi(t)+, t)$ for almost every $t$.

At this point, we give a list of properties of generalized characteristics of entropy solutions $u(x, t)$ for the balance laws (4). We shall make use of these Theorems and in particular of the No-crossing property of Theorem 3.3, to prove the $S B V_{l o c}$ regularity of $u(x, t)$. 
Theorem 3.2. Let $\chi($.$) be a generalized characteristic for (4), associated with the admis-$ sible solution $u$, which is genuine on $I=[a, b]$. Then there is a $C^{1}$ function $v$ defined on I such that:

$$
\begin{aligned}
& \text { 1. } u(\chi(a)-, a) \leq v(a) \leq u(\chi(a)+, a), \\
& \text { 2. } u(\chi(t)-, t)=v(t)=u(\chi(t)+, t) \text {, for } a<t<b \text {, } \\
& \text { 3. } u(\chi(b)-, b) \geq v(b) \geq u(\chi(b)+, b) .
\end{aligned}
$$

Furthermore, $(\chi(),. v()$.$) satisfy the classical characteristic equations$

$$
\left\{\begin{array}{l}
\dot{\chi}(t)=f_{u}(v(t), \chi(t), t) \\
\dot{v}(t)=-f_{x}(v(t), \chi(t), t)-g(v(t), \chi(t), t)
\end{array}\right.
$$

on $(a, b)$. In particular, $\chi$ is a $C^{1}$ function on $I$.

Remark 3.1. In [8], the generalized characteristic of Theorem 3.2 is assumed to be "shockfree". In here we state the Theorem for genuine characteristic because under an appropriate normalization, the notions of "shock-free" and "genuine" are equivalent. To conclude: we call shock-free a characteristic $\chi(t)$ such that $u(\chi(t)-, t)=u(\chi(t)+, t)$ for almost every $t$.

Theorem 3.3. Given a fixed point $(y, \tau) \in \mathbb{R} \times \mathbb{R}^{+}$we have that:

1. Through $(y, \tau)$ pass a minimal and a maximal backward characteristic denoted, respectively, by $\chi_{-}(t)$ and $\chi_{+}(t)$. The characteristics $\chi_{-}, \chi_{+}:[0, \tau] \rightarrow \mathbb{R}$ are genuine and are the solutions of the ODEs (14) with the following initial conditions: $\chi_{-}(\tau)=y$, $v_{-}(\tau)=u(y-, \tau)$ and $\chi_{+}(\tau)=y, v_{+}(\tau)=u(y+, \tau)$.

2. (No-crossing of characteristics). Two genuine characteristics may intersect only at their end points.

3. For $\tau>0$ through $(y, \tau)$ passes a unique forward characteristic. Furthermore, if $u(y+, \tau)<u(y-, \tau)$, then $u(\chi(t)+, t)<u(\chi(t)-, t)$ for all $t \in[\tau, \infty)$.

\section{Theorem 1.1}

In this part of the paper we analyze the regularity of the entropy solutions of the conservation laws (1). We recall that in [2] the flux function $f \in C^{2}$ was selected to be strictly convex and it was proved that entropy solutions are locally $S B V$. Here, in our first extension Theorem 1.1 we consider any flux function $f \in C^{2}$, which can change its convexity. Indeed, $f$ is selected such that $\#\left\{u_{i} \in \mathbb{R}: f^{\prime \prime}\left(u_{i}\right)=0\right\}$ is at most countable. 


\subsection{Strictly convex or concave flux function}

The first step in trying to extend Theorem 1.2 of [2] is to state the same result also for a conservation law with a strictly concave flux function, i.e. $f^{\prime \prime}<0$.

Lemma 4.1 (Strictly convex or concave flux). Let $f \in C^{2}(\mathbb{R})$ be a flux function with $\left|f^{\prime \prime}(u)\right|>0$. Let $u \in L^{\infty}(\Omega)$ be an entropy solution of the scalar conservation law (1). Then there exists a set $S \subset \mathbb{R}$ at most countable such that $\forall \tau \in \mathbb{R} \backslash S$ the following holds:

$$
u(., \tau) \in S B V_{l o c}\left(\Omega_{\tau}\right) \quad \text { with } \Omega_{\tau}:=\{x \in \mathbb{R}:(x, \tau) \in \Omega\} .
$$

Since the arguments are quite standard, we propose only a sketch of the proof of the above Lemma:

Proof. If $f^{\prime \prime}>0$, i.e. the flux function is strictly convex, the statement is exactly Theorem 1.2 of [2]. If $f^{\prime \prime}<0$, i.e. the flux function is strictly concave and we may prove the lemma directly using the convex case. The idea is simple: we reflect an entropy solution of the strictly convex case about the $t$-axis, to obtain an entropy solution of the related strictly concave problem. Let $f$ be strictly convex and assume that $u(x, t)$ is an entropy solution of (1). We define the coordinates transformation $\phi: \Omega \rightarrow \widetilde{\Omega}, \phi:(x, t) \mapsto(y, t)=(-x, t)$ and the candidate solution $\widetilde{u}(y, t):=u \circ \phi^{-1}(y, t)=u(-x, t)$ of the strictly concave problem. Then, we have:

$$
\begin{aligned}
D_{t} \widetilde{u}(y, t)+D_{y}[\widetilde{f}(\widetilde{u}(y, t))] & =D_{t} \widetilde{u}(y, t)-D_{y}[f(\widetilde{u}(y, t))]=D_{t} \widetilde{u}(-x, t)-\left(-D_{x}\right)[f(\widetilde{u}(-x, t))]= \\
& =D_{t} u(x, t)+D_{x}[f(u(x, t))]=0
\end{aligned}
$$

With $\tilde{f}=-f$ we denote a strictly concave flux. Moreover, for a point of $\Omega$ on a shock $x(t)$ with shock speed $\sigma=d x(t) / d t$, we must have that the Rankine-Hugoniot $\sigma\left[u^{R}-u^{L}\right]=$ $\left[f\left(u^{R}\right)-f\left(u^{L}\right)\right]$ and that the Lax-Entropy condition for a strictly convex flux $u^{L}>u^{R}$ are satisfied. We set $\widetilde{\sigma}=d(-x(t)) / d t=-\sigma, \widetilde{u}^{L}=u^{R}, \widetilde{u}^{R}=u^{L}$ and $\widetilde{f}=-f$, then after the reflection we obtain:

$$
\sigma\left[u^{R}-u^{L}\right]=\left[f\left(u^{R}\right)-f\left(u^{L}\right)\right] \Leftrightarrow \widetilde{\sigma}\left[\widetilde{u}^{R}-\widetilde{u}^{L}\right]=\left[\widetilde{f}\left(\widetilde{u}^{R}\right)-\widetilde{f}\left(\widetilde{u}^{L}\right)\right] .
$$

and $\widetilde{u}^{R}>\widetilde{u}^{L}$. This means that if $u(x, t)$ is an entropy solution of (1) for a strictly convex flux $f$, then $\widetilde{u}(y, t)=u(-x, t)$ is an entropy solution of the "correlate" conservation law with strictly concave flux $\widetilde{f}=-f$. In particular by the convex case and the definition of $\widetilde{u}$, there exists $\widetilde{S}=S \subset \mathbb{R}$ at most countable such that $\forall \tau \in \mathbb{R} \backslash \widetilde{S}$ the following holds:

$$
\widetilde{u}(., \tau) \in S B V_{l o c}\left(\widetilde{\Omega}_{\tau}\right) \quad \text { with } \widetilde{\Omega}_{\tau}:=\{x \in \mathbb{R}:(x, \tau) \in \widetilde{\Omega}\}
$$




\subsection{Proof of Theorem 1.1}

Step 1: Preliminary remarks.

Let us fix $(\xi, \tau) \in \Omega$ and $r$ such that $B_{r}(\xi, \tau) \subset \Omega$. Thanks to the finite speed of propagation, there exists a positive $\rho$ such that the values of $u$ in the ball $B_{\rho}(\xi, \tau)$ depend only on the values of $u$ on the segment $\{t=\tau-2 \rho\} \cap B_{r}(\xi, \tau)$. Thus, if we denote by $w$ the entropy solution of the Cauchy problem

$$
\begin{cases}D_{t} w(x, t)+D_{x}[f(w(x, t))]=0 & \text { for } t>\tau-2 \rho \\ w(x, \tau-2 \rho)=u(x, \tau-2 \rho) \mathbf{1}_{B_{r}(\xi, \tau)}(x, \tau-2 \rho) & \text { for every } x \in \mathbb{R}\end{cases}
$$

we get $w(x, t)=u(x, t)$ on $B_{\rho}(\xi, \tau)$. Moreover, note that $w(., t) \in B V$ for every $t>\tau-2 \rho$. Thus, it suffices to prove the main theorem under the assumptions that $\Omega=\{t>0\}$ and that $u(., 0)$ is a bounded compactly supported $B V$ function. By assumption, an entropy solution $u$ of the conservation laws (1) belongs to the space $B V(\Omega)$. Moreover, $u$ has a better structure than any 2-dimensional $B V$-function. Introducing some notation, we recall that if $u$ is an entropy solution of $(1)$, then $u_{\tau}():.=u(., \tau) \in B V\left(\Omega_{\tau}\right)$ for all $\tau$, where $\Omega_{\tau}:=\{x \in \mathbb{R}:(x, \tau) \in \Omega\}$. By Proposition 2.1 for any 1-dim $B V$ function the set of atoms $A$ coincides with the set of the discontinuous points; hence for all $\tau$ we introduce the sets of the jumps $J_{\tau}:=\left\{x \in \Omega_{\tau}: u_{\tau}^{L}(x) \neq u_{\tau}^{R}(x)\right\} \subset \mathbb{R}$ and $J:=\left\{(x, t) \in \Omega: x \in J_{t}\right\}$. By assumption the set $I_{f}:=\left\{u_{i} \in \mathbb{R}: f^{\prime \prime}\left(u_{i}\right)=0\right\}$, which contains all the inflection points of $f$, is at most countable. We conclude defining the set $F_{\tau}:=\left\{x \in \Omega_{\tau}: u_{\tau}(x)=u_{i}, u_{i} \in I_{f}\right\}$.

Step 2: Bad points have measure 0.

Using the above notation, we introduce the sets $C_{\tau}:=J_{\tau} \cup F_{\tau}$ for all $\tau$ and $C:=\{(x, t)$ : $\left.x \in C_{t}\right\}$, i.e. the sets of the "bad" points for which either $u_{\tau}($.$) has a jump or f^{\prime \prime}$ vanishes. For this two sets we state:

Claim 4.1. For any $\tau$ we have that $\left|D_{x}^{c} u_{\tau}\right|\left(C_{\tau}\right)=0$.

Proof. For every $\tau$ one has

$$
\left|D_{x}^{c} u_{\tau}\right|\left(C_{\tau}\right)=\left|D_{x}^{c} u_{\tau}\right|\left(J_{\tau} \cup F_{\tau}\right) \leq\left|D_{x}^{c} u_{\tau}\right|\left(J_{\tau}\right)+\left|D_{x}^{c} u_{\tau}\right|\left(F_{\tau}\right) .
$$

Observe that for all $\tau$ the Cantor part is zero on the jump sets $J_{\tau}$, since by (10):

$$
D_{x}^{c} u_{\tau}=D_{x} u_{\tau}\left\llcorner\left(S \backslash J_{\tau}\right) \quad \Rightarrow \quad D_{x}^{c} u_{\tau}\left(J_{\tau}\right)=0 \quad \Rightarrow \quad\left|D_{x}^{c} u_{\tau}\right|\left(J_{\tau}\right)=0 .\right.
$$

Using the second statement of Proposition 2.1, we show that even the second term of inequality (19) vanishes. Since $I_{f}$ is a countable set, we may rewrite this set as countable union of the sets $E_{i}:=\left\{u_{i}\right\}$, i.e. $I_{f}=\bigcup_{i} E_{i}$. It is clear that $\mathcal{L}^{1}\left(E_{i}\right)=0$ for each $i$ and that $F_{\tau}=\bigcup_{i} u_{\tau}^{-1}\left(E_{i}\right)=\bigcup_{i} u_{\tau}^{-1}\left(u_{i}\right)$. By Proposition 2.1, the Cantor part is zero on sets of the form $F_{\tau}=u_{\tau}^{-1}\left(I_{f}\right)$ with $\mathcal{L}^{1}\left(I_{f}\right)=0$. Hence, we obtain:

$$
\left|D_{x}^{c} u_{\tau}\right|\left(F_{\tau}\right)=\left|D_{x}^{c} u_{\tau}\right|\left(u_{\tau}^{-1}\left(I_{f}\right)\right)=0 .
$$

This concludes the proof of the claim, i.e. $\left|D_{x}^{c} u_{\tau}\right|\left(C_{\tau}\right)=0$. 


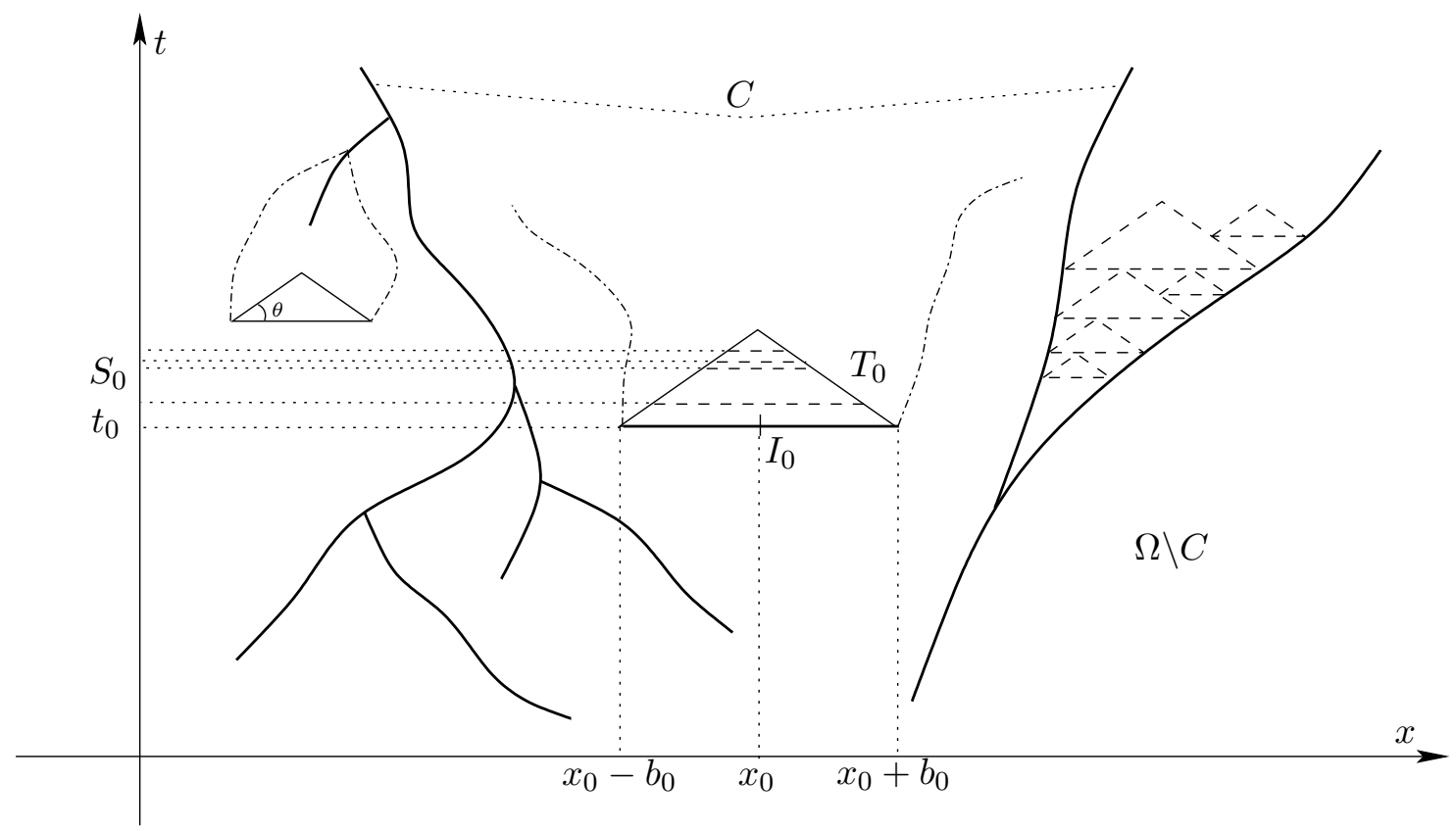

Figure 1: $\Omega \backslash C$ is covered by triangles..

Step 3: Locally, more precisely in a triangle, we reduce the problem to the cases with strictly convex or concave flux functions. By Lemma 4.1 the $S B V$-regularity follows.

Given any point $\left(x_{0}, t_{0}\right) \in \Omega \backslash C$ it is always possible to find a positive number $b_{0}=$ $b\left(x_{0}, t_{0}\right)>0$ and a positive $c_{0}=c\left(x_{0}, t_{0}\right)$, such that the following assertion holds:

$$
\left.\left|f^{\prime \prime}\left(u_{t_{0}}(x)\right)\right| \geq c_{0}>0 \text { for every } x \in I_{0}:=\right]-b_{0}+x_{0}, x_{0}+b_{0}[\text {. }
$$

Since $u_{t_{0}}$ is a continuous $B V$-function in $\Omega \backslash C_{t_{0}}$, we can also assume that there exists a positive $l_{0}\left(b_{0}\right)>0$ (which depends on $b_{0}$ ), such that $\left|u_{t_{0}}(x)-u_{i}\right| \geq l_{0}>0$ for all $x \in I_{0}$ and for any $u_{i} \in I_{f}$. This means that we may select $b_{0}>0$, such that the small variation of $u_{t_{0}}$ in $I_{0}$ allows to consider the Cauchy problem:

$$
\begin{cases}D_{t} w(x, t)+D_{x}\left[f_{0}(w(x, t))\right]=0 & \text { for } t>t_{0} ; \\ w\left(x, t_{0}\right)=u_{t_{0}}(x) \mathbf{1}_{I_{0}}\left(x, t_{0}\right) & \text { for every } x \in \mathbb{R},\end{cases}
$$

where the flux function $f_{0}$ is either strictly concave or strictly convex.

The finite speed of propagation of the characteristics permits to construct an isosceles triangle $T_{0}$ with base $I_{0}$, s.t. $w(x, t)=u(x, t)$ on the triangle $T_{0}$. Let $\theta\left(f,\|u\|_{L^{\infty}}\right)>0$ be the angle between the base $I_{0}$ and the diagonal segment. The angle $\theta$ depends only on $f$ and on $\|u\|_{L^{\infty}}$, since the slope of the maximal or the minimal characteristic of the problem defines this angle. We define the open triangle

$T_{0}:=T_{b_{0}}\left(x_{0}, t_{0}\right)=\left\{(x, t):\left|x-x_{0}\right|<b_{0}\right.$ and $\left.0<t-t_{0}<\tan \theta \cdot \min \left\{x-x_{0}+b_{0}, x_{0}+b_{0}-x\right\}\right\}$ 
By the maximum principle, the values of $u$ in $T_{0}$ are controlled by the values of $u_{t_{0}}$ on $I_{0}$. Moreover we may apply the statement of Lemma 4.1 on $T_{0}$, since the Cauchy problem (23) has either a strictly convex or a concave flux. In particular in our triangle $T_{0}$ there exists an at most countable set $S_{0}$ consisting of $\left.\tau \in\right] t_{0}, t_{0}+b_{0} \cdot \tan \theta[$ such that the solution is not $S B V\left(\Omega_{\tau} \cap T_{0}\right)$.

Step 4: Using Step 3, we construct a triangle for all the points of $\Omega \backslash C$. Let $B$ be the set of all points of $\Omega \backslash C$, which are contained in at least one of this triangle and divide $\Omega \backslash C$ into the two subsets $B$ and $C^{\prime}:=\Omega \backslash(C \cup B)$, i.e. $\Omega \backslash C=B \cup C^{\prime}$.

Claim 4.2. The set $\left\{\tau \in \mathbb{R}^{+}:\{t=\tau\} \cap C^{\prime} \neq \emptyset\right\}$ is at most countable.

Proof. Assume that $\left\{t:\{t=\tau\} \cap C^{\prime} \neq \emptyset\right\}$ is not countable. Let $\left\{P_{\alpha}\right\}=\left\{\left(x_{\alpha}, \tau_{\alpha}\right)\right\} \subset C^{\prime}$ be a subset of $C^{\prime} \subset \Omega \backslash C$, such that $\tau_{\alpha} \neq \tau_{\beta}$ whenever $\alpha \neq \beta$. Moreover, let $\left\{P_{\alpha}^{k}\right\}=\left\{\left(x_{\alpha}, \tau_{\alpha}\right)\right.$ : $\left.b\left(x_{\alpha}, \tau_{\alpha}\right) \geq 2^{-k}\right\} \subset\left\{P_{\alpha}\right\}$ be the subsets of the points, for which the base of the triangle is larger than $2^{-k+1}$, where $k \in \mathbb{N}$. By assumption there exists a fixed $K \in \mathbb{N}$ such that \# $P_{\alpha}^{K}$ is uncountable and thus the set $P_{\alpha}^{K}$ contains an accumulation point $p=\left(x_{p}, \tau_{p}\right)$. This implies that there exists a sequence $\left\{p_{j}\right\}_{j}:=\left\{\left(x_{j}, \tau_{j}\right)\right\}_{j \in \mathbb{N}} \subset P_{\alpha}^{K}$ of points in $C^{\prime}$, which converges to the accumulation point $p$. Moreover, any point $p_{j}$ of this sequence cannot be contained in the triangle $T_{p}:=T_{b\left(x_{p}, \tau_{p}\right)}\left(x_{p}, \tau_{p}\right)$, since by definition any point of $C^{\prime}$ cannot lie into a triangle. We then have that the sequence $\left\{p_{j}\right\}_{j}$ approaches $p$ from below, i.e. $\tau_{j}<\tau_{p}$ for every $j>J$ with $J$ big enough, and the triangles $T_{j}:=T_{b\left(x_{j}, \tau_{j}\right)}\left(x_{j}, \tau_{j}\right)$ have a base larger than $2^{-K+1}$ for each $j$. Thus, for a $J \in \mathbb{N}$ big enough the accumulation point $p$ belongs to the triangles $T_{j}$ for all $j>J$. This is a contradiction to $p \in C^{\prime}$.

Step 5: Cover with triangles.

By definition, every $(x, t) \in B$ lies into at least one triangle $T_{0}$ for a $\left(x_{0}, \tau_{0}\right) \in B$. The set $B$ is then covered by a family of triangles $\left\{T_{\alpha}\right\}_{\alpha}$. In particular we can find a countable subfamily of triangles $\left\{T_{i}\right\}_{i} \subset\left\{T_{\alpha}\right\}_{\alpha}$ which covers $B$, i.e. $B \subset \bigcup_{i} T_{i}$. We now divide $\Omega$ using the sets defined above:

$$
\Omega=(\Omega \backslash C) \cup C=B \cup C^{\prime} \cup C \subset \bigcup_{i} T_{i} \cup C^{\prime} \cup C .
$$

For every $\tau \in \mathbb{R}$ we have that:

- by Claim 4.2 the set $S_{C^{\prime}}=\left\{t:\{t=\tau\} \cap C^{\prime} \neq \emptyset\right\}$ is at most countable;

- by Lemma 4.1 for every $T_{i}$ the set $S_{i}:=\left\{t: u_{t} \notin S B V\left(\{t=\tau\} \cap T_{i}\right)\right\}$ is at most countable. Thus, for every time $\tau \notin S:=S_{C^{\prime}} \cup\left(\bigcup_{i} S_{i}\right)$ we have the following inequality:

$$
\begin{aligned}
\left|D_{x}^{c} u_{\tau}\right|(\Omega \cap\{t=\tau\}) & \leq\left|D_{x}^{c} u_{\tau}\right|\left(\bigcup_{i} T_{i} \cap\{t=\tau\}\right)+\left|D_{x}^{c} u_{\tau}\right|(C \cap\{t=\tau\}) \leq \\
& \leq \sum_{i}\left|D_{x}^{c} u_{\tau}\right|\left(T_{i} \cap\{t=\tau\}\right)+\left|D_{x}^{c} u_{\tau}\right|(C \cap\{t=\tau\})=0 .
\end{aligned}
$$


All terms in the sum vanish by Lemma 4.1 and the second term is equal to zero by Claim 4.1. Letting $\Omega_{\tau}=\Omega \cap\{t=\tau\}$ we have shown that $\forall \tau \in \mathbb{R} \backslash S$ the following holds:

$$
u_{\tau}(.)=u(., \tau) \in S B V_{l o c}\left(\Omega_{\tau}\right) .
$$

\section{Proof of Theorem 1.2}

As far as we know the estimate (5) of Theorem 1.2 has never been proved. In this section we then use the generalized characteristics to obtain that:

Proposition 5.1. Let $f, g$ be as in the statement of Theorem 1.2, in particular in any compact set $K \subset \Omega$ there exist constants $C_{1}, C_{2}>0$ with

$$
\|D f\|_{L^{\infty}(K)},\left\|D^{2} f\right\|_{L^{\infty}(K)},\|D g\|_{L^{\infty}(K)} \leq C_{1}
$$

and

$$
f_{u u}(.) \geq C_{2}>0
$$

If $u(x, t)$ is an entropy solution of the balance equation (4), then for all $\varepsilon>0$ there exists a constant $C_{3}:=C_{3}\left(C_{1}, C_{2}, \varepsilon\right)>0$ with

$$
u([x+z]+, t)-u(x-, t) \leq C_{3} z, \quad(z>0)
$$

for every fixed $t \in[\varepsilon, 1]$ and for all $x \in \mathbb{R}$ with $(x, t),(x+z, t) \in K$.

\subsection{Preparatory for the proof of Proposition 5.1}

Let $t \in[\varepsilon, 1]$ be fixed and take $x \in \mathbb{R}$ and $z>0$ with $(x, t),(x+z, t) \in K$. Let us denote by $\chi_{-}(s)$ the minimal backward characteristic passing through $(x, t)$ and by $\chi_{+}(s)$ the maximal backward characteristic passing through $(x+z, t)$, (instead of $\chi_{-}(s ; x, t)$ and $\left.\chi_{+}(s ; x+z, t)\right)$. Rewriting the ODEs (14) related to the genuine characteristics for $\chi_{-}(s), \chi_{+}(s):[\varepsilon, t] \rightarrow \mathbb{R}$ we obtain

$$
\left\{\begin{array}{l}
\dot{\chi}_{ \pm}(s)=f_{u}\left(v_{ \pm}(s), \chi_{ \pm}(s), s\right) \\
\dot{v}_{ \pm}(s)=-f_{x}\left(v_{ \pm}(s), \chi_{ \pm}(s), s\right)-g\left(v_{ \pm}(s), \chi_{ \pm}(s), s\right)
\end{array}\right.
$$

with

$$
\left\{\begin{array}{l}
\chi_{-}(t)=x \\
\chi_{+}(t)=x+z \\
v_{-}(t)=u(x-, t)=: u^{-} \\
v_{+}(t)=u([x+z]+, t)=: u^{+}
\end{array}\right.
$$

where by the admissible condition (11), $u^{-}>u^{+}$. We recall that by the no crossing property of Theorem 3.3 of the genuine characteristics, the distance between the two curves is positive i.e. $\chi_{+}(s)>\chi_{-}(s)$ for every $s \in[\varepsilon, t]$. 


\subsection{One Technical Lemma}

For the proof of our theorem we will use the following technical lemma:

Lemma 5.1. Assume that there exists a constant $C_{3}>0$ with

$$
u([x+z]+, t)-u(x-, t) \geq C_{3} z, \quad(z>0) .
$$

Then there exists $\delta:=\delta\left(C_{1}, C_{2}, \varepsilon\right)$ with $0<\delta<\varepsilon$ and such that

$$
v_{+}(s)-v_{-}(s) \geq \frac{C_{3}}{16} z, \quad \forall s \in[t-\delta, t] .
$$

Proof. We subdivide the proof into two steps:

Step 1: We claim that:

Claim 5.1. Let $C_{3}>0$ be sufficiently big and assume that there exists a $\delta>0$ such that inequality (30) holds. Then, we have

$$
\dot{\chi}_{+}(s)-\dot{\chi}_{-}(s)>0, \quad \forall s \in[t-\delta, t]
$$

If our claim were not true, then it would exists $\tau \in[t-\delta, t]$ such that

$$
\left\{\begin{array}{l}
\left.\left.\dot{\chi}_{+}(s)-\dot{\chi}_{-}(s)>0, \quad \forall s \in\right] \tau, t\right] \\
\dot{\chi}_{+}(\tau)-\dot{\chi}_{-}(\tau)=0 .
\end{array}\right.
$$

By the equation of the characteristics (77) it follows that:

$$
\dot{\chi}_{+}(\tau)-\dot{\chi}_{-}(\tau)=f_{u}\left(v_{+}(\tau), \chi_{+}(\tau), \tau\right)-f_{u}\left(v_{-}(\tau), \chi_{-}(\tau), \tau\right)=: U(\tau)+W(\tau)
$$

The two terms in (33) are defined as

$$
U(\tau):=f_{u}\left(v_{+}(\tau), \chi_{+}(\tau), \tau\right)-f_{u}\left(v_{-}(\tau), \chi_{+}(\tau), \tau\right) \geq C_{2}\left(v_{+}(\tau)-v_{-}(\tau)\right),
$$

and

$$
W(\tau):=f_{u}\left(v_{-}(\tau), \chi_{+}(\tau), \tau\right)-f_{u}\left(v_{-}(\tau), \chi_{-}(\tau), \tau\right) \geq-C_{1}\left|\chi_{+}(\tau)-\chi_{-}(\tau)\right|
$$

Thus by $(33),(34),(35)$ and $(30),(32)$ we obtain that

$$
\begin{aligned}
\dot{\chi}_{+}(\tau)-\dot{\chi}_{-}(\tau) & \geq C_{2}\left(v_{+}(\tau)-v_{-}(\tau)\right)-C_{1}\left|\chi_{+}(\tau)-\chi_{-}(\tau)\right| \\
& \geq C_{2} \frac{C_{3}}{16} z-C_{1} z=\left(\frac{C_{2} C_{3}}{16}-C_{1}\right) z
\end{aligned}
$$

Clearly in the last inequality we have used the bounds (24) and (25). So if we choose $C_{3}>\frac{16 C_{1}}{C_{2}}$, then

$$
\dot{\chi}_{+}(\tau)-\dot{\chi}_{-}(\tau)>0
$$


and this is in contradiction with the definition of $\tau$.

Step 2: Let us define the time

$$
t_{0}:=\sup _{s \in[t-\delta, t]}\left\{s: v_{+}(s)-v_{-}(s)<\frac{C_{3}}{16} z\right\}
$$

If $t_{0}<t-\eta$ for any $\eta>0$ then we can conclude the proof, because we can trivially select $\delta=\eta$ in (30). Otherwise from Step 1 we know that

$$
0<\chi_{+}(s)-\chi_{-}(s) \leq z, \quad \forall s \in\left[t_{0}, t\right] .
$$

Moreover, by the definition of $t_{0}$ we know even that

$$
v_{+}\left(t_{0}\right)-v_{-}\left(t_{0}\right)=\frac{C_{3}}{16} z .
$$

Using the equations of the characteristics (77), the bounds (24), (25) and computing as in Step 1, we can state that for every $s \in\left[t_{0}, t\right]$ it holds that:

$$
\begin{aligned}
\left|\dot{v}_{+}(s)-\dot{v}_{-}(s)\right| & \leq 2 C_{1}\left|v_{+}(s)-v_{-}(s)\right|+2 C_{1}\left|\chi_{+}(s)-\chi_{-}(s)\right| \\
& \stackrel{(38)}{\leq} 2 C_{1}\left(\left|v_{+}(s)-v_{-}(s)\right|+z\right)
\end{aligned}
$$

Putting $E(s):=\left|v_{+}(s)-v_{-}(s)\right|+z$ from the last inequality we get to:

$$
\dot{E}(s) \leq 2 C_{1} E(s)
$$

Thus, by Gronwall's Lemma we have that for every $s \in\left[t_{0}, t\right]$ :

$$
E(s) \leq e^{2 C_{1}\left(s-t_{0}\right)} E\left(t_{0}\right)
$$

Choosing $s=t$ we obtain:

$$
\left(C_{3} z+z\right) \stackrel{(29)}{\leq} E(t) \stackrel{(42)}{\leq} e^{2 C_{1}\left(t-t_{0}\right)} E\left(t_{0}\right) \stackrel{(39)}{=} e^{2 C_{1}\left(t-t_{0}\right)}\left(\frac{C_{3}}{16} z+z\right)
$$

Thus,

$$
t-t_{0} \geq \frac{1}{2 C_{1}} \log \left(\frac{16 C_{3}+16}{C_{3}+16}\right)
$$

and in particular if $C_{3}$ is big enough we conclude that $t-t_{0}>0$. This means that there exists $\delta>0$ such that $t-\delta \in\left[t_{0}, t\right]$ and the inequality (30) is satisfied. 


\subsection{Proof of Proposition 5.1}

Case 1: Trivially, if it holds

$$
u([x+z]+, t)-u(x-, t) \leq C_{3} z, \quad(z>0)
$$

our proposition is true.

Case 2: Otherwise, we can choose an $\alpha>C_{3}$ with

$$
\frac{u([x+z]+, t)-u(x-, t)}{z}=\alpha, \quad(z>0) .
$$

By Lemma 5.1 there exists $0<\delta<\varepsilon$ such that for every $s \in[t-\delta, t]$ we have the following two estimates:

$$
\begin{aligned}
& 0<\chi_{+}(s)-\chi_{-}(s) \leq z, \\
& v_{+}(s)-v_{-}(s) \geq \frac{\alpha z}{16} .
\end{aligned}
$$

Now, using the same techniques and bounds as above we compute:

$$
\begin{aligned}
\chi_{+}(t)-\chi_{-}(t) & =\int_{t-\delta}^{t} f_{u}\left(v_{+}(s), \chi_{+}(s), s\right)-f_{u}\left(v_{-}(s), \chi_{-}(s), s\right) d s+\chi_{+}(t-\delta)-\chi_{-}(t-\delta) \\
& \geq \int_{t-\delta}^{t} f_{u}\left(v_{+}(s), \chi_{+}(s), s\right)-f_{u}\left(v_{-}(s), \chi_{-}(s), s\right) d s \\
& \geq C_{2} \frac{\alpha \delta}{16} z-\int_{t-\delta}^{t} C_{1}\left|\chi_{+}(s)-\chi_{-}(s)\right| d s \geq C_{2} \frac{\alpha \delta}{16} z-\delta z C_{1}
\end{aligned}
$$

Since $z=\chi_{+}(t)-\chi_{-}(t)$ we rewrite $(49)$ as

$$
z\left(1+C_{1} \delta\right) \geq C_{2} \frac{\alpha \delta}{16} z \quad \Rightarrow \quad \alpha \leq \frac{16\left(1+C_{1} \delta\right)}{\delta C_{2}}
$$

and then

$$
u([x+z]+, t)-u(x-, t) \leq \frac{16\left(1+C_{1} \delta\right)}{\delta C_{2}} z, \quad(z>0)
$$

This conclude the proof.

\section{$6 \quad$ Preparatory tools for the proof of Theorem 1.3}

In the geometric proof of the main Theorem in [2] the characteristics of the entropy solutions of the scalar conservation laws (1) played a fundamental role. Using the good and simple structure of these characteristics it was possible to define a monotone geometric functional, which jumps when a Cantor part appears in the solution. One of the key Lemmas was the No-crossing-Proposition (see Proposition 2.5 of [2]), which implies that two different 
characteristics $\chi_{1}:\left[0, \tau_{1}\right] \rightarrow \mathbb{R}, \chi_{2}:\left[0, \tau_{2}\right] \rightarrow \mathbb{R}$ cannot cross for all $t \in\left(0, \max \left\{\tau_{1}, \tau_{2}\right\}\right)$. The generalized characteristics of a balance laws (4) with source term $g$ are in general no more straight lines, but by Theorem 3.3 we know that the No-crossing property still holds for two distinct genuine characteristics. By the way, for every point $(y, \tau) \in \mathbb{R} \times \mathbb{R}^{+}$ the minimal and maximal backward characteristics are genuine and then the No-crossing property is assured. This suggests us that the construction proposed in [2] still works even for equations (4) and that we can try to restate the main steps of the original proofs. In particular, a geometric approach to our problem make sense and in this section we shall then introduce a geometric functional defined using the generalized characteristics. We begin by giving some preliminary definitions and propositions, which are inspired to those presented in [2]:

Definition 6.1. (Characteristic cones and bases). Let $\tau>0$. For $\theta \in[0, \tau]$ the backward characteristic cone $C_{y, \tau}^{\theta}$ emanating from $y \in S_{\tau}:=\{x \in \mathbb{R}: u(x-, \tau) \neq u(x+, \tau)\}$ is defined as the open "triangle" having

$$
(y, \tau),\left(\chi_{-}(\theta ; y, \tau), \theta\right),\left(\chi_{+}(\theta ; y, \tau), \theta\right)
$$

as vertices. The base of a characteristic cone at time $\theta \in[0, \tau]$ is defined as the open interval:

$$
\left.I_{y, \tau}^{\theta}:=\right] \chi_{-}(\theta ; y, \tau), \chi_{+}(\theta ; y, \tau)[.
$$

We note that the characteristic cones are confined by minimal and maximal backward characteristics, which are genuine. Then, due to the No-crossing property of genuine characteristics two different cones $C_{y_{1}, \tau_{1}}^{\theta}$ and $C_{y_{2}, \tau_{2}}^{\theta}$ (or two different bases $I_{y_{1}, \tau_{1}}^{\theta}$ and $I_{y_{2}, \tau_{2}}^{\theta}$ ) are either one contained in the other or disjoint. We define also

$$
C_{\tau}^{\theta}:=\bigcup_{y \in S_{\tau}} C_{y, \tau}^{\theta} \quad \text { and } \quad I_{\tau}^{\theta}:=\bigcup_{y \in S_{\tau}} I_{y, \tau}^{\theta}
$$

It is known that entropy solutions $u(x, t)$ of $(4)$ are $B V$ functions. Moreover, for every $t$ the function $u(., t)$ is also $B V$ on $\mathbb{R}$ and then by (10) we can split $D_{x} u(., t)$ in three mutually singular parts:

$$
D_{x} u(., t)=D_{x}^{a} u(., t)+D_{x}^{j} u(., t)+D_{x}^{c} u(., t)
$$

For convenience we denote by $\mu_{t}:=D_{x}^{c} u(., t)$ the Cantor part and by $\nu_{t}:=D_{x}^{j} u(., t)$ the jump part. Inequality (11) implies that the singular measures $\mu_{t}$ and $\nu_{t}$ are both nonpositive. We recall also the semi-monotonicity of $u(., t)$ that gives

$$
u(y+, t)-u(x-, t)=D u(., t)([x, y]) \quad \text { whenever } x<y .
$$

Finally we state three technical lemmas, which are to compare with estimates (3.4), (3.10) and Lemma 3.2 of [2]. 


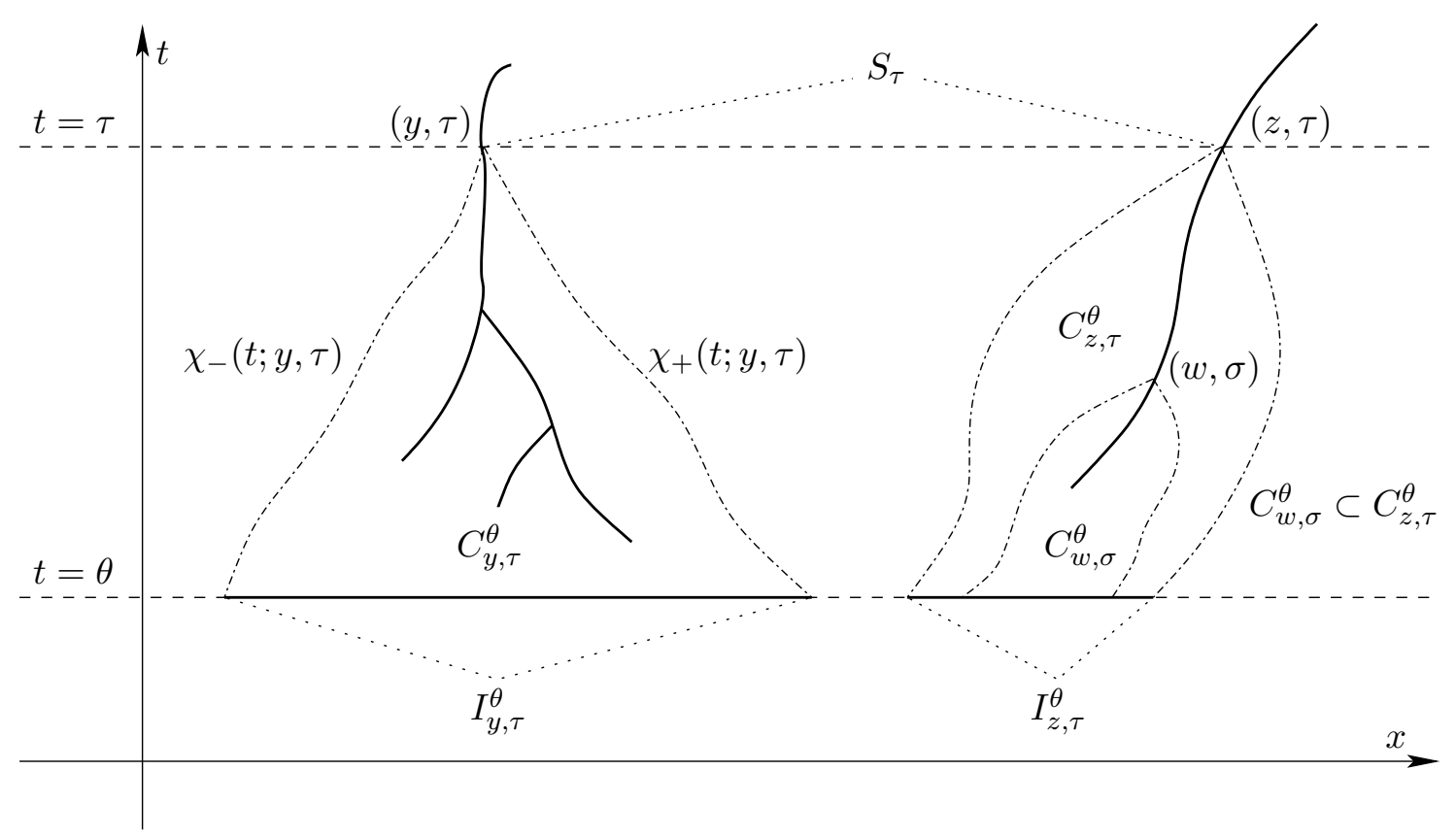

Figure 2: Characteristic cones and bases.

Lemma 6.1. Let $\tau>0$. If $y \in S_{\tau}$, then for all $\theta \in[0, \tau]$ there exists a positive constant $c_{j}$ such that

$$
\mathcal{L}^{1}\left(I_{y, \tau}^{\theta}\right)=\chi_{+}(\theta ; y, \tau)-\chi_{-}(\theta ; y, \tau) \leq-c_{j} \nu_{\tau}(\{y\})
$$

Lemma 6.2. Let $\tau_{0}>0$. Then for $\mu_{\tau_{0}}$-a.e. $x$ there exists $\eta:=\eta\left(x, \tau_{0}, \tau\right)>0$ such that

$$
] x-\eta, x+\eta\left[\subset I_{\tau}^{\tau_{0}} \quad \text { for } \tau>\tau_{0} .\right.
$$

Definition 6.2. We denote by $E$ the set of $x$ 's for which Lemma 6.2 applies and such that

$$
\lim _{\eta \downarrow 0} \frac{\left|D u\left(., \tau_{0}\right)-\mu_{\tau_{0}}\right|([x-\eta, x+\eta])}{-\mu_{\tau_{0}}([x-\eta, x+\eta])}=0 .
$$

The Besicovitch differentiation theorem gives that $\mu_{\tau_{0}}(\mathbb{R} \backslash E)=0$ and with (54) we have for every $x \in E$ that

$$
\lim _{\eta \downarrow 0} \frac{u\left((x-\eta)-, \tau_{0}\right)-u\left((x+\eta)+, \tau_{0}\right)}{-\mu_{\tau_{0}}([x-\eta, x+\eta])}=1 .
$$

Lemma 6.3. Let $\tau_{0}>0$. For every $x \in E$, for every $\eta>0$ such that $x \pm \eta \notin S_{\tau_{0}}$ and for all $\theta \in\left(0, \tau_{0}\right]$, there exists a positive constant $c_{c}(\theta)$ such that

$$
\mathcal{L}^{1}\left(J_{x, \eta}^{\theta}\right)=\chi_{+}\left(\theta ; x+\eta, \tau_{0}\right)-\chi_{-}\left(\theta ; x-\eta, \tau_{0}\right) \geq-c_{c}(\theta) \mu_{\tau_{0}}([x-\eta, x+\eta])
$$

where $\left.J_{x, \eta}^{\theta}:=\right] \chi_{-}\left(\theta ; x-\eta, \tau_{0}\right), \chi_{+}\left(\theta ; x+\eta, \tau_{0}\right)[$.

Note that the proofs of these lemmas are listed in Chapter 9 and that to get these results we will use the estimate of Theorem 1.2. 


\section{$7 \quad$ Proof of Theorem 1.3}

Step 1: Preliminary remarks.

Let us fix $(\xi, \tau) \in \Omega$ and a radius $r$ such that the ball $B_{r}(\xi, \tau) \subset \Omega$. Thanks to the finite speed of propagation, there exists a positive $\rho$ such that the values of $u(x, t)$ in the ball $B_{\rho}(\xi, \tau)$ depend only on the values of $u$ on the segment $\{t=\tau-2 \rho\} \cap B_{r}(\xi, \tau)$. If we write $w$ for the entropy solution of the problem

$$
\begin{cases}D_{t} w(x, t)+D_{x}[f(w(x, t), x, t)]+g(w(x, t), x, t)=0, & \text { for } t>\tau-2 \rho \\ w(x, \tau-2 \rho)=u(x, \tau-2 \rho) \mathbf{1}_{B_{r}(\xi, \tau)}(x, \tau-2 \rho), & \text { for every } x \in \mathbb{R}\end{cases}
$$

we get that $w=u$ on $B_{\rho}(\xi, \tau)$. We also note that $w(., t) \in B V(\mathbb{R})$ for every $t>\tau-2 \rho$. Thus it suffices to prove the theorem under the additional assumptions that $\Omega=\{t>0\}$ and that $u(., 0)$ is a bounded compactly supported $B V$ function. Since the function $u(., 0)$ has compact support, we know that there exist constants $R$ and $c_{R}$ such that the support of $u(., t)$ is contained in $\{|x| \leq R\}$ and

$$
|D u(., t)|(\mathbb{R}) \leq c_{R}
$$

Step 2: Definition of the geometric functional $F^{\theta}(t)$.

For a fixed $\theta>0$ we define the functional $F^{\theta}:\left[\theta, \infty\left[\rightarrow \mathbb{R}^{+}\right.\right.$as follows

$$
F^{\theta}(t):=\mathcal{L}^{1}\left(I_{t}^{\theta}\right)=\sum_{y \in S_{t}} \mathcal{L}^{1}\left(I_{y, t}^{\theta}\right)
$$

where the second equality holds by the No-crossing property of the characteristics. Geometrically the functional $F^{\theta}(t)$ measures the total length of the bases at time $\theta$ of all characteristic cones contained in $C_{t}^{\theta}$. With Lemma 6.1 we observe that this functional is bounded from above:

$$
F^{\theta}(t) \leq-c_{j} \nu_{t}(\mathbb{R}) \leq c_{j}|D u(., t)|(\mathbb{R}) \leq c_{j} c_{R} \quad \text { for every } t \in[\theta, T] .
$$

Moreover, this geometric functional is nondecreasing. This is a consequence of the fact that for every $\theta \leq t_{1} \leq t_{2}$ we have that $I_{t_{1}}^{\theta} \subset I_{t_{2}}^{\theta}$. Assuming that there exists $x \in S_{t_{1}}$ such that $x \in\left(I_{t_{1}}^{t_{2}}\right)^{c}$, then the shock $s(t)$ passing through $\left(x, t_{1}\right)$ would be not entirely contained in the characteristic cones $C_{t_{2}}^{t_{1}}$. But by definition of characteristic cones and the No-crossing property, every shock in $\mathbb{R} \times\left[t_{1}, t_{2}\right]$ is entirely contained in $C_{t_{2}}^{t_{1}}$. Therefore, $S_{t_{1}} \subset I_{t_{2}}^{t_{1}}$ and again due to the No-crossing property we conclude that $I_{t_{1}}^{\theta} \subset I_{t_{2}}^{\theta}$. We state

Lemma 7.1. For fixed $T \geq \theta>0$ the functional $F^{\theta}:[\theta, T] \rightarrow \mathbb{R}^{+}$is nondecreasing and bounded from above.

One of the key remarks of the proof given in [2] was the connection between the jumps of the geometric functional and the Cantor parts created in the solution. We can try to describe this geometric process as follows: when a Cantor part is created in the solution 
at time $\tau_{0}$, this part is transformed after an infinitesimal time into several small jumps. The sum of the measures of these jumps give a contribution big enough to make the geometrical functional jump at $\tau_{0}$. Due to the similar construction of our functional $F^{\theta}($. and the original functional defined in [2], we can utilize the same idea here. This motivates a first reduction of our problem to the following Lemma:

Lemma 7.2. For any integer $k$ we have

$$
\tau_{0} \geq \frac{T}{k}>\theta \text { and } \mu_{\tau_{0}}(\mathbb{R}) \leq-\frac{1}{k} \quad \Rightarrow \quad F^{\theta}\left(\tau_{0}+\right) \geq F^{\theta}\left(\tau_{0}\right)+c_{F}(\theta)
$$

where $c_{F}(\theta)$ is a strictly positive constant which depends on $\|u\|_{\infty}, T, k, f, g$ and on the choice of $\theta>0$.

Clearly, Lemma 7.1 and Lemma 7.2 imply that all sets

$$
\left\{\tau \in \left[\frac{T}{k}, T\left[: \mu_{\tau}(\mathbb{R}) \leq-\frac{1}{k}\right\}\right.\right.
$$

are finite.

Step 3: Proof of Lemma 7.2.

In this step we shall make use of the three technical Lemmas 6.1, 6.2 and 6.3. We fix $\tau>\tau_{0} \geq T / k \geq \theta$. Let $x \in E$, where $E$ is the set defined by Lemma 6.1. Consequently to Lemma 6.3 , for $\eta>0$ small enough we have that

$$
\mathcal{L}^{1}\left(J_{x, \eta}^{\theta}\right) \geq-c_{c}(\theta) \mu_{\tau_{0}}([x-\eta, x+\eta])
$$

where $\left.J_{x, \eta}^{\theta}:=\right] \chi_{-}\left(\theta ; x-\eta, \tau_{0}\right), \chi_{+}\left(\theta ; x+\eta, \tau_{0}\right)[$. By the No-crossing property of characteristics we know that $J_{x, \eta}^{\theta}$ can only intersect the bases of the cones $I_{y, \tau_{0}}^{\theta}$ emanating from a point $y \in[x-\eta, x+\eta]$, so that recalling Lemma 6.1 it follows that

$$
\mathcal{L}^{1}\left(J_{x, \eta}^{\theta} \cap I_{\tau_{0}}^{\theta}\right)=\sum_{y \in S_{\tau_{0}} \cap[x-\eta, x+\eta]} \mathcal{L}^{1}\left(I_{y, \tau_{0}}^{\theta}\right) \leq-c_{j} \nu_{\tau_{0}}([x-\eta, x+\eta]) .
$$

Combining (66) and (67) we find that for any $x \in E$ we have that

$$
\begin{aligned}
\mathcal{L}^{1}\left(J_{x, \eta}^{\theta} \backslash I_{\tau_{0}}^{\theta}\right) & \geq-c_{c}(\theta) \mu_{\tau_{0}}([x-\eta, x+\eta])+c_{j} \nu_{\tau_{0}}([x-\eta, x+\eta]) \geq \\
& \geq-c_{c}(\theta) \mu_{\tau_{0}}([x-\eta, x+\eta])-c_{j}\left|\nu_{\tau_{0}}\right|([x-\eta, x+\eta]) .
\end{aligned}
$$

Finally, invoking the Besicovitch differentiation theorem and in particular (57), we obtain

$$
\mathcal{L}^{1}\left(J_{x, \eta}^{\theta} \backslash I_{\tau_{0}}^{\theta}\right) \geq-\frac{c_{c}(\theta)}{2} \mu_{\tau_{0}}([x-\eta, x+\eta]),
$$

provided that $\eta$ is small enough. Using the Besicovitch covering lemma, we can cover $\mu_{\tau_{0}}$-a.e. $E$ with pairwise disjoint intervals $K_{j, \tau_{0}}^{\theta}:=\left[x_{j}-\eta_{j}, x_{j}+\eta_{j}\right]$ such that (69) and the conclusion of Lemma 6.2 both hold for $x=x_{j}$ and $\eta=\eta_{j}$. Thanks to the No-crossing 
property all the intervals $J_{x_{j}, \eta_{j}}^{\theta}$ are pairwise disjoint and recalling Lemma 6.2 we note that all these sets are contained in $I_{\tau}^{\theta}$. In Lemma 7.2 we assumed that $-\mu_{\tau_{0}}(\mathbb{R}) \geq 1 / k$. Then for all $\theta>0$ the estimates above imply:

$$
\begin{aligned}
F^{\theta}(\tau)-F^{\theta}\left(\tau_{0}\right) & \geq \sum_{j} \mathcal{L}^{1}\left(J_{x_{j}, \eta_{j}}^{\theta} \backslash I_{\tau_{0}}^{\theta}\right) \geq-\sum_{j} \frac{c_{c}(\theta)}{2} \mu_{\tau_{0}}\left(\left[x_{j}-\eta_{j}, x_{j}+\eta_{j}\right]\right) \geq \\
& \geq-\frac{c_{c}(\theta)}{2} \mu_{\tau_{0}}(E)=-\frac{c_{c}(\theta)}{2} \mu_{\tau_{0}}(\mathbb{R}) \geq \frac{c_{c}(\theta)}{2 k}=: c_{F}(\theta) .
\end{aligned}
$$

Step 4: The end of the proof.

In Step 3 we have shown that for any fixed $\theta>0$ the interval $(\theta, T]$ contains a set $H_{\theta}$, which is at most countable and such that for every $\tau \in(\theta, T] \backslash H_{\theta}$ the following holds:

$$
u(., \tau) \in S B V_{l o c}\left(\Omega_{\tau, \theta}\right) \quad \text { with } \Omega_{\tau, \theta}:=\{x \in \mathbb{R}:(x, \tau) \in \Omega, \tau \in(\theta, T]\} .
$$

Now, we consider the sequence $\left(\theta_{n}\right)_{n \in \mathbb{N}}:=T / 2^{n}$ and we define the set $S$ of the main Theorem 1.3 as the countable union of the countable sets $H_{\theta_{n}} \subset\left(\theta_{n}, T\right]$, which is again countable:

$$
S:=H_{0}=\bigcup_{n \in \mathbb{N}} H_{\frac{T}{2^{n}}}
$$

This concludes the proof.

\section{Theorem 1.4 and Corollary 1.1}

The proof of Theorem 1.4 combines the ideas of Theorem 1.1 and Theorem 1.3. We repeat it for the reader's convenience.

Proof. Step 1: [Strictly concave flux.] As in the proof of Theorem 1.1 let us first show that there exists a locally $S B V$ entropy solution for the problem with a strictly concave flux. By Theorem 1.3 we know that if $f(u(x, t), x, t)$ is strictly convex in $u$ and $C^{2}$, the equation

$$
D_{t} u(x, t)+D_{x}[f(u(x, t), x, t)]+g(u(x, t), x, t)=0
$$

has a locally $S B V$ entropy solution $u(x, t)$. Since $f_{1}(u(x, t), x, t):=f(u(x, t),-x, t)$ is again $C^{2}$ and strictly convex in $u$ and $g_{1}(u(x, t), x, t):=g(u(x, t),-x, t)$ is $C^{1}$, the solution $u_{1}(x, t)$ of

$$
D_{t} u_{1}(x, t)+D_{x}\left[f_{1}\left(u_{1}(x, t), x, t\right)\right]+g_{1}\left(u_{1}(x, t), x, t\right)=0
$$

is again a $S B V_{\text {loc }}$ function. Next we consider the coordinates transformation $\phi: \Omega \rightarrow$ $\widetilde{\Omega}, \phi:(x, t) \mapsto(y, t)=(-x, t)$ and we put $\widetilde{f}:=-f, \widetilde{g}:=g$. Then we have for $\widetilde{u}(y, t):=$ 


$$
\begin{aligned}
& u_{1} \circ \phi^{-1}(y, t)=u_{1}(-x, t): \\
& \qquad \begin{aligned}
D_{t} \widetilde{u}(y, t) & +D_{y}[\widetilde{f}(\widetilde{u}(y, t), y, t)]+\widetilde{g}(\widetilde{u}(y, t), y, t)= \\
& =D_{t} \widetilde{u}(y, t)-D_{y}[f(\widetilde{u}(y, t), y, t)]+g(\widetilde{u}(y, t), y, t)= \\
& =D_{t} \widetilde{u}(-x, t)-\left(-D_{x}\right)[f(\widetilde{u}(-x, t),-x, t)]+g(\widetilde{u}(-x, t),-x, t)= \\
& =D_{t} u_{1}(x, t)+D_{x}\left[f\left(u_{1}(x, t),-x, t\right)\right]+g\left(u_{1}(x, t),-x, t\right)= \\
& =D_{t} u_{1}(x, t)+D_{x}\left[f_{1}\left(u_{1}(x, t), x, t\right)\right]+g_{1}\left(u_{1}(x, t), x, t\right)=0 .
\end{aligned}
\end{aligned}
$$

Thus $\widetilde{u}$ is a solution of a balance law with strictly concave flux function and since $u_{1}$ is locally $S B V$ this implies that also $\widetilde{u} \in S B V_{l o c}$. Moreover, $u_{1}$ is an entropy solution of (74) and since we have found the solution $\widetilde{u}$ reflecting $u_{1}$ about the $t$-axis, as in the proof of Lemma 4.1, we can show that even for $\widetilde{u}$ the entropy and the Rankine-Hugoniot conditions hold.

Step 2: $\left[u(x, t) \in S B V_{l o c}\left(\Omega_{\tau}\right)\right.$.] At this point we can repeat the construction given in the proof of Theorem 1.1. Due to the small variation of an entropy solution we can restrict the solution $u(x, t)$ on triangles $T_{j}$ 's, where the flux function $f$ is either strictly convex or strictly concave. Recalling that $C$ is the set of points $\left(x_{i}, t_{i}\right)$ where the solution has jumps or has a values such that $f_{u u}\left(u\left(x_{i}, t_{i}\right), x_{i}, t_{i}\right)=0$, we cover $\Omega \backslash C$ with a countable family of triangles $T_{j}$. For the bad points not contained in one triangle or points of $C$, we can restate Claim 4.2 and 4.1 . We conclude that the entropy solution $u(x, t)$ is locally $S B V$ on $\Omega_{\tau}$ for every $\tau \in \mathbb{R} \backslash S$.

Step 3: $\left[u \in S B V_{l o c}(\Omega)\right.$.] The slicing theory says that the 2-dimensional Cantor part of the derivative $D_{x} u(x, t)$ can be recovered from the corresponding 1-dimensional part. By Theorem 3.108 of [1] we have:

$$
D_{x}^{c} u(x, t)=\int \mathcal{L}^{1}\left\llcorner\Omega_{t} \otimes D^{c} u(., t) d t\right.
$$

where $\Omega_{t}$ is the projection of $\Omega$ on $\{x=0\} \times \mathbb{R}^{+}$. Since by Step 2 the Cantor part is $D^{c} u(., t)=0$ for every $t \notin S$ and $S$ is at most countable, then also the two dimensional Cantor part $D_{x}^{c} u(x, t)$ vanishes. With the Vol'perts chain rule (see Theorem 3.96 of [1]) and equation (4) we get that $D_{t}^{c} u(x, t)=0$. Finally we have obtained that $u \in S B V_{l o c}(\Omega)$.

Next, we use Theorem 1.3 to prove corollary 1.1.

Proof. We differentiate the equation (9) by $x$ (in the sense of distributions):

$$
D_{x} w_{t}(x, t)+D_{x} H\left(w_{x}(x, t), x, t\right)=0 \quad \Leftrightarrow \quad D_{t} w_{x}(x, t)+D_{x} H\left(w_{x}(x, t), x, t\right)=0 .
$$

Letting $u(x, t)=w_{x}(x, t)$ and $H(u(x, t), x, t)=f(u(x, t), x, t)$, this is exactly the balance equation of Theorem 1.3, i.e. $u(x, t)=w_{x}(x, t)$ is $S B V_{l o c}\left(\Omega_{\tau}\right)$ for every $\tau \in \mathbb{R} \backslash S$, where $S$ is at most countable. As in Step 3 of the proof of Theorem 1.4, it follows that $u(x, t) \in$ $S B V_{l o c}(\Omega)$. 


\section{Proofs of the three technical lemmas}

\subsection{Proof of Lemma 6.1}

Let $\tau>0$ and $y \in S_{\tau}$. To simplify the notation, we denote the minimal and the maximal backward characteristics starting from $(y, \tau)$ by $\chi_{-}(t)$ and $\chi_{+}(t)$ instead of $\chi_{-}(t ; y, \tau)$ and $\chi_{+}(t ; y, \tau)$. Rewriting the ODEs $(14)$ related to the genuine characteristics for $\chi_{-}(t), \chi_{+}(t)$ : $[0, \tau] \rightarrow \mathbb{R}$ we obtain

$$
\left\{\begin{array}{l}
\dot{\chi}_{ \pm}(t)=f_{u}\left(v_{ \pm}(t), \chi_{ \pm}(t), t\right) \\
\dot{v}_{ \pm}(t)=-f_{x}\left(v_{ \pm}(t), \chi_{ \pm}(t), t\right)-g\left(v_{ \pm}(t), \chi_{ \pm}(t), t\right)
\end{array}\right.
$$

with

$$
\left\{\begin{array}{l}
\chi_{-}(\tau)=y=\chi_{+}(\tau) \\
v_{-}(\tau)=u(y-, \tau)=: u^{-} \\
v_{+}(\tau)=u(y+, \tau)=: u^{+}
\end{array}\right.
$$

where by the admissible condition (11), $u^{-}>u^{+}$and $-\nu_{\tau}(\{y\})=u^{-}-u^{+}$. Now we change the variable in equations (77) putting $s=\tau-t$ :

$$
\left\{\begin{array}{l}
\dot{\psi}_{ \pm}(s)=-f_{u}\left(\omega_{ \pm}(s), \psi_{ \pm}(s), \tau-s\right) \\
\dot{\omega}_{ \pm}(s)=f_{x}\left(\omega_{ \pm}(s), \psi_{ \pm}(s), \tau-s\right)+g\left(\omega_{ \pm}(s), \psi_{ \pm}(s), \tau-s\right)
\end{array}\right.
$$

where $\psi_{ \pm}(s):=\chi_{ \pm}(\tau-s)$ and $\omega_{ \pm}(s):=v_{ \pm}(\tau-s)$. Then, using (79) we have

$$
\begin{aligned}
\frac{d}{d s}\left|\psi_{-}(s)-\psi_{+}(s)\right| & \leq\left|\dot{\psi}_{-}(s)-\dot{\psi}_{+}(s)\right|=\left|f_{u}\left(\omega_{-}(s), \psi_{-}(s), \tau-s\right)-f_{u}\left(\omega_{+}(s), \psi_{+}(s), \tau-s\right)\right| \\
& \leq \underbrace{\left\|D^{2} f\right\|_{L^{\infty}(K)}}_{:=c_{1}}\left(\left|\omega_{-}(s)-\omega_{+}(s)\right|+\left|\psi_{-}(s)-\psi_{+}(s)\right|\right) .
\end{aligned}
$$

Repeating the computations also for $\omega_{ \pm}(s)$, we find that:

$$
\begin{aligned}
\frac{d}{d s}\left|\omega_{-}(s)-\omega_{+}(s)\right| \leq & \left|f_{x}\left(\omega_{-}(s), \psi_{-}(s), \tau-s\right)-f_{x}\left(\omega_{+}(s), \psi_{+}(s), \tau-s\right)\right|+ \\
& +\left|g\left(\omega_{-}(s), \psi_{-}(s), \tau-s\right)-g\left(\omega_{+}(s), \psi_{+}(s), \tau-s\right)\right| \leq \\
\leq & \underbrace{\left[\left\|D^{2} f\right\|_{L^{\infty}(K)}+\|D g\|_{\left.L^{\infty}(K)\right]}\right]}_{:=c_{2}}\left(\left|\omega_{-}(s)-\omega_{+}(s)\right|+\left|\psi_{-}(s)-\psi_{+}(s)\right|\right)
\end{aligned}
$$

If we choose a compact set

$$
K:=\left[-\|u\|_{\infty},\|u\|_{\infty}\right] \times\left[\min _{t \in[0, \tau]} \chi_{-}(t), \max _{t \in[0, \tau]} \chi_{+}(t)\right] \times[0, \tau]
$$

then the above constants $c_{1}$ and $c_{2}$ are positive and finite. Inequalities (80) and (81) together give

$$
\frac{d}{d s}\left(\left|\psi_{-}(s)-\psi_{+}(s)\right|+\left|\omega_{-}(s)-\omega_{+}(s)\right|\right) \leq c_{3}\left(\left|\psi_{-}(s)-\psi_{+}(s)\right|+\left|\omega_{-}(s)-\omega_{+}(s)\right|\right)
$$


where $c_{3}$ is again a positive constant depending only on $\left\|D^{2} f\right\|_{L^{\infty}(K)}$ and $\|D g\|_{L^{\infty}(K)}$. By Gronwall's lemma and (83) we get

$$
\left|\psi_{-}(s)-\psi_{+}(s)\right|+\left|\omega_{-}(s)-\omega_{+}(s)\right| \leq e^{c_{3} s}\left(\left|\psi_{-}(0)-\psi_{+}(0)\right|+\left|\omega_{-}(0)-\omega_{+}(0)\right|\right) .
$$

Now, we put $s=\vartheta \in[0, \tau]$ into (84) to find the following inequality:

$$
\begin{aligned}
\left|\chi_{-}(\tau-\vartheta)-\chi_{+}(\tau-\vartheta)\right| & =\left|\psi_{-}(\vartheta)-\psi_{+}(\vartheta)\right| \leq\left|\psi_{-}(\vartheta)-\psi_{+}(\vartheta)\right|+\left|\omega_{-}(\vartheta)-\omega_{+}(\vartheta)\right| \leq \\
& \leq \underbrace{e^{c_{3} \tau}}_{:=c_{\tau}}\left(\left|\psi_{-}(0)-\psi_{+}(0)\right|+\left|\omega_{-}(0)-\omega_{+}(0)\right|\right)= \\
& =c_{\tau}\left(\left|\chi_{-}(\tau)-\chi_{+}(\tau)\right|+\left|v_{-}(\tau)-v_{+}(\tau)\right|\right) .
\end{aligned}
$$

If we set $\theta=\tau-\vartheta$, then by (78) we conclude:

$$
\begin{aligned}
\chi_{+}(\theta)-\chi_{-}(\theta) & =\left|\chi_{-}(\theta)-\chi_{+}(\theta)\right| \leq c_{\tau}(\underbrace{\left|\chi_{-}(\tau)-\chi_{+}(\tau)\right|}_{=0}+\underbrace{\left|v_{-}(\tau)-v_{+}(\tau)\right|}_{=\left|u^{-}-u^{+}\right|}) \\
& \leq c_{\tau}\left(u^{-}-u^{+}\right)=-c_{\tau} \nu_{\tau}(\{y\}) .
\end{aligned}
$$

\subsection{Proof of Lemma 6.2}

We prove that the conclusion of the lemma holds for any $x$ which satisfy the following conditions:

$$
x \notin S_{\tau_{0}} \quad \text { and } \quad \lim _{\eta \downarrow 0} \frac{u\left(x+\eta, \tau_{0}\right)-u\left(x-\eta, \tau_{0}\right)}{2 \eta}=-\infty .
$$

By the Besicovitch differentiation theorem on intervals, the measure $\mu_{\tau_{0}}$ is concentrated on $E$. In our proof we fix $\tau>\tau_{0}$ and $x$ such that (87) holds and our goal is to show that for $\eta$ small enough $\left.\left\{\tau_{0}\right\} \times\right] x-\eta, x+\eta\left[\subset I_{\tau}^{\tau_{0}}\right.$. To prove that the point $x$ is contained in $I_{\tau}^{\tau_{0}}$, we consider all the possible cases:

$$
\begin{aligned}
I & : x \notin \overline{I_{\tau}^{\tau_{0}}} \\
I I & : x \in \partial\left(\overline{I_{\tau}^{\tau_{0}}}\right) \\
I I I & : \exists \eta>0 \text { s.t. }(] x-\eta, x+\eta\left[\cap I_{\tau}^{\tau_{0}}\right)^{c}=\{x\} \\
I V & : x \in I_{\tau}^{\tau_{0}}
\end{aligned}
$$

and in particular we will show that in the first three cases we obtain a contradiction. Then, by IV the point $x$ is in $I_{\tau}^{\tau_{0}}$ and since $I_{\tau}^{\tau_{0}}$ is open there exists $\eta>0$ such that $\left.\left\{\tau_{0}\right\} \times\right] x-\eta, x+\eta\left[\subset I_{\tau}^{\tau_{0}}\right.$.

The property (87) may be rewritten prescribing that for every positive $\alpha>\bar{\alpha}>0$, there exists $\bar{\eta}>0$ such that for all $0<\eta<\bar{\eta}$ holds

$$
u\left(x-\eta, \tau_{0}\right)-u\left(x+\eta, \tau_{0}\right)>\alpha 2 \eta>\bar{\alpha} 2 \eta>0 .
$$

The positive number $\bar{\alpha}$ will be chosen later, more precisely we will define this constant at the end of case I. 
Case I: Since in this case the distance $\operatorname{dist}\left(x, \overline{I_{\tau}^{\tau_{0}}}\right)$ is strictly positive there exists $\eta>0$ small enough such that $0<2 \eta<\bar{\eta}$ and $] x-\eta, x+\eta\left[\cap \overline{I_{\tau}^{\tau_{0}}}=\emptyset\right.$. Consequently to the definition of a characteristic cone, we observe that all possible shocks created before $\tau$ are contained in $C_{\tau}^{\tau_{0}}$ and thus, two different shocks starting at $x_{1}, x_{2} \notin \overline{I_{\tau}^{\tau_{0}}}$, where $x_{1} \neq x_{2}$, cannot cross for all $t \in\left[\tau_{0}, \tau\right]$. By the way, also the two characteristics passing trough $\left(x-\eta, \tau_{0}\right)$ and $\left(x+\eta, \tau_{0}\right)$, which are genuine, cannot cross in $\left[\tau_{0}, \tau\right]$. If we denote by $\chi_{1}(t)$ and $\chi_{2}(t)$, respectively, the two characteristics $\chi\left(t ; x-\eta, \tau_{0}\right)$ and $\chi\left(t ; x+\eta, \tau_{0}\right)$, this implies $\chi_{1}(t)<\chi_{2}(t)$ for every $t \in\left[\tau_{0}, \tau\right]$. This conclusion is contradicted by the next claim.

Claim 9.1. If $x$ is a point such that $\exists \eta>0$ with $] x-\eta, x+\eta\left[\cap \overline{I_{\tau}^{\tau_{0}}}=\emptyset\right.$ and (88) holds then

$$
\chi_{1}(\tau)-\chi_{2}(\tau)>0 .
$$

Proof. Integrating the ODEs of the characteristics (14) we obtain:

$$
\begin{aligned}
& \int_{\tau_{0}}^{\tau} \dot{\chi}(t) d t=\int_{\tau_{0}}^{\tau} f_{u}(v(t), \chi(t), t) d t \\
& \chi(\tau)=\chi\left(\tau_{0}\right)+\int_{\tau_{0}}^{\tau} f_{u}(v(t), \chi(t), t) d t
\end{aligned}
$$

and

$$
\begin{aligned}
& \int_{\tau_{0}}^{t} \dot{v}(s) d s=-\int_{\tau_{0}}^{t} f_{x}(v(s), \chi(s), s)+g(v(s), \chi(s), s) d s \\
& v(t)=v\left(\tau_{0}\right)-\int_{\tau_{0}}^{t} f_{x}(v(s), \chi(s), s)+g(v(s), \chi(s), s) d s .
\end{aligned}
$$

Substituting $\chi_{1}$ and $\chi_{2}$ to $\chi$ in (90) and subtracting the corresponding equations, by the mean value theorem there exists $\xi$ such that:

$$
\begin{aligned}
\chi_{1}(\tau)-\chi_{2}(\tau) & =\chi_{1}\left(\tau_{0}\right)-\chi_{2}\left(\tau_{0}\right)+\int_{\tau_{0}}^{\tau} f_{u}\left(v_{1}(t), \chi_{1}(t), t\right)-f_{u}\left(v_{2}(t), \chi_{2}(t), t\right) d t= \\
& =-2 \eta+\int_{\tau_{0}}^{\tau} f_{u u}(\xi)\left(v_{1}(t)-v_{2}(t)\right)+f_{u x}(\xi)\left(\chi_{1}(t)-\chi_{2}(t)\right) d t
\end{aligned}
$$

holds. We observe that by the finite speed of the characteristics, the distance between $\chi_{1}(t)$ and $\chi_{2}(t)$ for every $t \in\left[\tau_{0}, \tau\right]$ is controlled by the distance of the characteristics at the time $\tau_{0}$ :

$$
\left|\chi_{1}(t)-\chi_{2}(t)\right| \leq C_{\chi}\left|\chi_{1}\left(\tau_{0}\right)-\chi_{2}\left(\tau_{0}\right)\right|=C_{\chi} 2 \eta \quad \forall t \in\left[\tau_{0}, \tau\right]
$$

where $C_{\chi}$ is a positive constant. Thus by (92) and (93):

$$
\begin{aligned}
-2 \eta+\int_{\tau_{0}}^{\tau} f_{u u}(\xi)\left(v_{1}(t)-v_{2}(t)\right) d t & =\chi_{1}(\tau)-\chi_{2}(\tau)-\int_{\tau_{0}}^{\tau} f_{u x}(\xi)\left(\chi_{1}(t)-\chi_{2}(t)\right) d t \leq \\
& \leq \chi_{1}(\tau)-\chi_{2}(\tau)+\int_{\tau_{0}}^{\tau}\left|f_{u x}(\xi)\left(\chi_{1}(t)-\chi_{2}(t)\right)\right| d t \leq \\
& \leq \chi_{1}(\tau)-\chi_{2}(\tau)+\underbrace{\left(\tau-\tau_{0}\right)\left\|D^{2} f\right\|_{L^{\infty}(K)} C_{\chi}}_{:=\widetilde{C}_{f}} 2 \eta
\end{aligned}
$$


where $K$ is a compact set, for instance

$$
K:=\left[-\|u\|_{\infty},\|u\|_{\infty}\right] \times\left[\min _{t \in\left[\tau_{0}, \tau\right]}\left\{\chi_{1}(t), \chi_{2}(t)\right\}, \max _{t \in\left[\tau_{0}, \tau\right]}\left\{\chi_{1}(t), \chi_{2}(t)\right\}\right] \times\left[\tau_{0}, \tau\right] .
$$

Next we employ (91) to estimate the rest of (94). For every $t \in\left[\tau_{0}, \tau\right]$ we have that:

$$
\begin{aligned}
v_{1}(t)-v_{2}(t)= & v_{1}\left(\tau_{0}\right)-v_{2}\left(\tau_{0}\right)+\underbrace{\int_{\tau_{0}}^{t} f_{x}\left(v_{2}(s), \chi_{2}(s), s\right)-f_{x}\left(v_{1}(s), \chi_{1}(s), s\right) d s}_{:=A(t)}+ \\
& +\underbrace{\int_{\tau_{0}}^{t} g\left(v_{2}(s), \chi_{2}(s), s\right)-g\left(v_{1}(s), \chi_{1}(s), s\right) d s}_{=: B(t)} .
\end{aligned}
$$

By the mean value theorem for two appropriate $\sigma_{1}$ and $\sigma_{2}$ the terms $A(t)$ and $B(t)$ are equal to:

$$
\begin{aligned}
& A(t)=\int_{\tau_{0}}^{t} f_{u x}\left(\sigma_{1}\right)\left(v_{2}(s)-v_{1}(s)\right)+f_{x x}\left(\sigma_{1}\right)\left(\chi_{2}(s)-\chi_{1}(s)\right) d s \\
& B(t)=\int_{\tau_{0}}^{t} g_{u}\left(\sigma_{2}\right)\left(v_{2}(s)-v_{1}(s)\right)+g_{x}\left(\sigma_{2}\right)\left(\chi_{2}(s)-\chi_{1}(s)\right) d s
\end{aligned}
$$

and then

$$
\begin{aligned}
|A(t)+B(t)| & \leq \int_{\tau_{0}}^{t}\left|\left(f_{u x}\left(\sigma_{1}\right)+g_{u}\left(\sigma_{2}\right)\right)\left(v_{2}(s)-v_{1}(s)\right)+\left(f_{x x}\left(\sigma_{1}\right)+g_{x}\left(\sigma_{2}\right)\right)\left(\chi_{2}(s)-\chi_{1}(s)\right)\right| d s \leq \\
& \leq \int_{\tau_{0}}^{t}(\underbrace{\left\|D^{2} f\right\|_{L^{\infty}(K)}+\|D g\|_{L^{\infty}(K)}}_{:=C_{f, g}})\left(\left|v_{2}(s)-v_{1}(s)\right|+\left|\chi_{2}(s)-\chi_{1}(s)\right|\right) d s \leq \\
& \stackrel{(5)}{\leq} C_{f, g} \int_{\tau_{0}}^{t}(C+1)\left|\chi_{2}(s)-\chi_{1}(s)\right| d s \stackrel{(93)}{\leq} C_{f, g} \int_{\tau_{0}}^{t}(C+1) C_{\chi} 2 \eta d s \leq \\
& \leq C_{f, g}(C+1)\left[\tau-\tau_{0}\right] C_{\chi} 2 \eta=: \tilde{C}_{f, g} 2 \eta
\end{aligned}
$$

where the resulting constant $\tilde{C}_{f, g}$ is positive. Combining the inequality (99) with (96) and (94) we obtain:

$$
\begin{aligned}
& -2 \eta\left(1+\widetilde{C}_{f}\right)+\int_{\tau_{0}}^{\tau} f_{u u}(\xi)\left(v_{1}\left(\tau_{0}\right)-v_{2}\left(\tau_{0}\right)\right) d t \leq \int_{\tau_{0}}^{\tau}\left|f_{u u}(\xi)[A(t)+B(t)]\right| d t+\chi_{1}(\tau)-\chi_{2}(\tau) \\
& \stackrel{(99)}{\leq} \underbrace{\left(\tau-\tau_{0}\right)\left\|D^{2} f\right\|_{L^{\infty}(K)} \widetilde{C}_{f, g}}_{:=\widetilde{C}_{f, g}} 2 \eta+\chi_{1}(\tau)-\chi_{2}(\tau) .
\end{aligned}
$$


Finally, we take a better form for the last estimate

$$
\begin{aligned}
\chi_{1}(\tau)-\chi_{2}(\tau) & \geq-2 \eta\left(1+\widetilde{C}_{f}+\widehat{C}_{f, g}\right)+\int_{\tau_{0}}^{\tau} f_{u u}(\xi)\left(v_{1}\left(\tau_{0}\right)-v_{2}\left(\tau_{0}\right)\right) d t= \\
& =-2 \eta \underbrace{\left(1+\widetilde{C}_{f}+\widehat{C}_{f, g}\right)}_{>0}+\int_{\tau_{0}}^{f_{\geq \gamma>0}^{f_{u u}(\xi)}} \underbrace{\left(u\left(x-\eta, \tau_{0}\right)-u\left(x+\eta, \tau_{0}\right)\right)}_{>0 \text { for } \eta \text { small enough by }(88)} d t
\end{aligned}
$$

where $\gamma>0$ is the constant related to the strictly convexity of $f(u, x, t)$ in $u$, i.e.

$$
f_{u u}(u, x, t) \geq \gamma>0
$$

for fixed $(x, t)$. Now, we put

$$
\bar{\alpha}=\frac{1+\widetilde{C}_{f}+\widehat{C}_{f, g}}{\left(\tau-\tau_{0}\right) \gamma}>0 .
$$

We note that $\bar{\alpha}$ is positive and it depends only on $\left\|D^{2} f\right\|_{L^{\infty}(K)},\|D g\|_{L^{\infty}(K)}, \tau_{0}, \tau$ and $\gamma$. Recalling (88) and (100) for $\bar{\alpha}>0$, there exists $\bar{\eta}>0$ such that for every small $\eta$ with $0<\eta<\bar{\eta}$ we have

$$
\chi_{1}(\tau)-\chi_{2}(\tau)>-2 \eta\left(1+\widetilde{C}_{f}+\widehat{C}_{f, g}\right)+2 \eta \bar{\alpha}\left(\tau-\tau_{0}\right) \gamma \stackrel{(101)}{=} 0
$$

Case II: If $x$ belongs to $\partial\left(\overline{I_{\tau_{0}}}\right)$, then one of the two characteristics $\chi\left(t ; x-\eta, \tau_{0}\right)$ or $\chi\left(t ; x+\eta, \tau_{0}\right)$ is not contained in $C_{\tau_{0}}^{\tau}$. Moreover, the characteristic $\chi\left(t ; x, \tau_{0}\right)$ is a boundary curve of the characteristic cone $C_{\tau_{0}}^{\tau}$ and so it is either a minimal or a maximal backward characteristic. Repeating similar computations as in case I it is possible to show that if (87) holds, either $\chi\left(t ; x-\eta, \tau_{0}\right)$ or $\chi\left(t ; x+\eta, \tau_{0}\right)$ will cross with $\chi\left(t ; x, \tau_{0}\right)$ for a $\left.t \in\right] \tau_{0}, \tau[$. Recalling again the No-crossing property of genuine characteristic, we get a contradiction. Case III: By Theorem 3.3 for every $\tau_{0}>0$ trough $\left(x, \tau_{0}\right)$ passes a unique forward characteristic. Consequently case III is to discard.

Case IV: In view of the contradictions obtained in the previous cases, the last possible case must be true. In particular $x \in I_{\tau_{0}}^{\tau}$ and since $I_{\tau_{0}}^{\tau}$ is open there exists a $\eta>0$ small enough such that

$$
] x-\eta, x+\eta\left[\subset I_{\tau_{0}}^{\tau}\right.
$$

for $\tau>\tau_{0}$.

Remark 9.1. We remark that in [2] Lemma 6.2 was proved using the Hopf-Lax formula. Here we have proposed a more geometrical construction, which make use of the properties of generalized characteristics. This change of strategy is also motivated by the fact that for system of conservation laws the Hopf-Lax does not exists, whereas there is a suitable concept of generalized characteristics (see [8]). 


\subsection{Proof of Lemma 6.3}

Let $\tau_{0}>0, x \in E$ and $\eta>0$ such that $x \pm \eta \notin S_{\tau_{0}}$. To simplify the notation we write $\chi_{-}(t)$ and $\chi_{+}(t)$ instead of $\chi_{-}\left(t ; x-\eta, \tau_{0}\right)$ and $\chi_{+}\left(t ; x+\eta, \tau_{0}\right)$. Our aim is to show that for all $\left.\theta \in] 0, \tau_{0}\right]$ there exists a positive constant $c_{c}(\theta)$ such that

$$
\chi_{+}(\theta)-\chi_{-}(\theta) \geq-c_{c}(\theta) \mu_{\tau_{0}}([x-\eta, x+\eta])
$$

holds. By Besicovitch differentiation theorem (58) we have

$$
\begin{aligned}
-c \mu_{\tau_{0}}([x-\eta, x+\eta]) & \leq u\left(x-\eta, \tau_{0}\right)-u\left(x+\eta, \tau_{0}\right) \leq \\
& \leq\left|u\left(x-\eta, \tau_{0}\right)-u\left(x+\eta, \tau_{0}\right)\right|+\left|\chi_{-}\left(\tau_{0}\right)-\chi_{+}\left(\tau_{0}\right)\right|= \\
& =\left|v_{-}\left(\tau_{0}\right)-v_{+}\left(\tau_{0}\right)\right|+\left|\chi_{-}\left(\tau_{0}\right)-\chi_{+}\left(\tau_{0}\right)\right| .
\end{aligned}
$$

Next we derive analogous estimates as in the proof of Lemma 6.1. Now we fix $\tau \in] 0, \tau_{0}[$ and we change the variable in equations of the characteristics (77) putting $s=t-\tau$ :

$$
\left\{\begin{array}{l}
\dot{\psi_{ \pm}}(s)=f_{u}\left(\omega_{ \pm}(s), \psi_{ \pm}(s), \tau+s\right) \\
\dot{\omega_{ \pm}}(s)=-f_{x}\left(\omega_{ \pm}(s), \psi_{ \pm}(s), \tau+s\right)-g\left(\omega_{ \pm}(s), \psi_{ \pm}(s), \tau+s\right)
\end{array}\right.
$$

where $\psi_{ \pm}(s):=\chi_{ \pm}(\tau+s)$ and $\omega_{ \pm}(s):=v_{ \pm}(\tau+s)$. Using Gronwall's Lemma, on the compact set $K$ defined in (82) there exists a positive constant $c_{K}$, which depends only on $\left\|D^{2} f\right\|_{L^{\infty}(K)}$ and $\|D g\|_{L^{\infty}(K)}$, such that

$$
\left|\psi_{-}(s)-\psi_{+}(s)\right|+\left|\omega_{-}(s)-\omega_{+}(s)\right| \leq e^{c_{K} s}\left(\left|\psi_{-}(0)-\psi_{+}(0)\right|+\left|\omega_{-}(0)-\omega_{+}(0)\right|\right) .
$$

We insert $s=\tau_{0}-\tau$ in the inequality above and by $\chi_{ \pm}\left(\tau_{0}\right)=\psi_{ \pm}\left(\tau_{0}-\tau\right), \chi_{ \pm}(\tau)=\psi_{ \pm}(0)$ and $v_{ \pm}\left(\tau_{0}\right)=\omega_{ \pm}\left(\tau_{0}-\tau\right), v_{ \pm}(\tau)=\omega_{ \pm}(0)$, it follows that

$$
\left|\chi_{-}\left(\tau_{0}\right)-\chi_{+}\left(\tau_{0}\right)\right|+\left|v_{-}\left(\tau_{0}\right)-v_{+}\left(\tau_{0}\right)\right| \leq \underbrace{e^{c_{K} \tau_{0}}}_{:=\bar{c}}\left(\left|\chi_{-}(\tau)-\chi_{+}(\tau)\right|+\left|v_{-}(\tau)-v_{+}(\tau)\right|\right)
$$

Thus, with (103) we get:

$$
\begin{aligned}
-c \mu_{\tau_{0}}([x-\eta, x+\eta]) & \leq \bar{c}\left(\left|\chi_{-}(\tau)-\chi_{+}(\tau)\right|+\left|v_{-}(\tau)-v_{+}(\tau)\right|\right) \leq \\
& \stackrel{(5)}{\leq} \bar{c}\left(\left|\chi_{-}(\tau)-\chi_{+}(\tau)\right|+C\left|\chi_{-}(\tau)-\chi_{+}(\tau)\right|\right) \leq \\
& \leq(\bar{c}+C)\left|\chi_{-}(\tau)-\chi_{+}(\tau)\right|
\end{aligned}
$$

We have proved, that for all $\theta \in\left(0, \tau_{0}\right]$ there exists a positive constant $c_{c}(\theta)$ depending only on $\left\|D^{2} f\right\|_{L^{\infty}(K)},\|D g\|_{L^{\infty}(K)}, \tau_{0}$ and $\theta$ such that:

$$
\chi_{+}(\theta)-\chi_{-}(\theta)=\left|\chi_{-}(\theta)-\chi_{+}(\theta)\right| \geq-c_{c}(\theta) \mu_{\tau_{0}}([x-\eta, x+\eta]) .
$$




\section{Acknowledgments}

I'm deeply grateful to Professor Camillo De Lellis for his encouragement, support and cooperation to this work.

\section{References}

[1] L.Ambrosio, N.Fusco \& D.Pallara: Functions of bounded variation and free discontinuity problems. Oxford Mathematical Monographs, 2000.

[2] L.Ambrosio \& C.De Lellis: A note on admissible solutions of 1d scalar conservation laws and 2d Hamilton-Jacobi equations. J. Hyperbolic Diff. Equ., 31(4), 2004, pp. 813-826.

[3] A.Bressan: Hyperbolic Systems of Conservation Laws: The One-Dimensional Cauchy Problem., Oxford University Press, 2000.

[4] P.Cannarsa, A.Mennucci \& C.Sinestrari: Regularity results for solutions of a class of Hamilton-Jacobi Equations, Arch. Rational Mech. Anal. 140, 1997, pp. $197-223$.

[5] P.Cannarsa \& C.Sinestrari: Semiconcave Functions, Hamilton-Jacobi Equations, and Optimal Control, Birkhäuser, Boston, 2004.

[6] C.M.Dafermos: Characteristics in hyperbolic conservation laws. A study of the structure and the asymptotic behaviour of solutions, Nonlinear Analysis and Mechanics: Heriot-Watt Symposium, Vol I, ed. R.J. Knops. London: Pitman, 1977, pp. 1-58.

[7] C.M.Dafermos: Generalized Characteristics and the structure of solutions of hyperbolic conservation laws. Indiana U.Math. J. 26, 1977, pp.1097-1119.

[8] C.M.Dafermos: Hyperbolic conservation laws in continuum physics. Grundlehren der matematischen Wissenschaften. Vol.325. Second Edition. Springer Verlag, Heidelberg, 2005.

[9] L.C.Evans: Partial differential equations. Graduate Studie in Mathmathics 319, AMS, 1991.

[10] A.F.Filippov: Differential equations with discountinuous right-hand side, Mat. Sb. 51, 1960, pp.99-128. (English tr.: Amer. Math. Soc. Transl. Ser., 2, 42, 1964, 199-231).

[11] K.Hvistendahl Karlsen, K.-A. Lie \& N.H.Risebro: A front tracking method for conservation laws with boundary conditions. Hyperbolic problems: theory, numerics, applications, Vol. I (Zürich,1998), Internat. Ser. Numer. Math. 129, Birkhäuser, Basel, 1999, pp.493-502. 
(Roger Robyr) Institut FÜR MAthematik, Universität ZÜRICH, WinterthurERSTRASSE 190, CH-8057 ZÜRICH, SwITZERLAND

E-mail: roger.robyr@math.unizh.ch 